\title{
Continuous Phase-Modulated Halftones
}

\author{
Basak Oztan, Student Member, IEEE, and Gaurav Sharma, Senior Member, IEEE
}

\begin{abstract}
A generalization of periodic clustered-dot halftones is proposed, wherein the phase of the halftone spots is modulated using a secondary signal. The process is accomplished by using an analytic halftone threshold function that allows halftones to be generated with controlled phase variation in different regions of the printed page. The method can also be used to modulate the screen frequency, albeit with additional constraints. Visible artifacts are minimized/eliminated by ensuring the continuity of the modulation in phase. Limitations and capabilities of the method are analyzed through a quantitative model. The technique can be exploited for two applications that are presented in this paper: a) embedding watermarks in the halftone image by encoding information in phase or in frequency and b) modulating the screen frequency according to the frequency content of the continuous tone image in order to improve spatial and tonal rendering. Experimental performance is demonstrated for both applications.
\end{abstract}

Index Terms-Clustered-dot halftones, continuous phase modulation, halftone watermarking, spatial/tonal rendering.

\section{INTRODUCTION}

$\mathbf{H}$ ALFTONING is a common method to reproduce a continuous tone (contone) image on bi-level printers or displays such that at normal viewing distances, the halftone image conveys the same visual impression as the contone image. A large number of halftoning methods have been proposed for the rendering of halftone images on bi-level printers [3], [4]. Because of its stability and reproducibility, clustered-dot halftoning [5] is the primary halftoning technique for electrophotography and lithography, which are the two primary methods of high-volume printing.

Digital clustered-dot halftones are commonly generated by comparing each pixel in the contone image against a corresponding threshold. The thresholds for each of the pixels in the image are obtained via periodic replication of a small set of thresholds defined over a 2-D tiling region called a halftone cell. Digital halftones generated by this method mimic the conventional analog halftones produced by the photographic screening

Manuscript received October 09, 2008; revised June 30, 2009. First published July 24, 2009; current version published November 13, 2009. Parts of this work were presented in IEEE ICASSP 2006 [1] and in IEEE ICIP 2006 [2]. This work was supported in part by a gift from the Xerox Foundation and in part by a grant from New York State Office of Science, Technology, and Academic Research (NYSTAR) through the Center for Electronic Imaging Systems (CEIS) of University of Rochester. The associate editor coordinating the review of this manuscript and approving it for publication was Dr. Gabriel Marcu.

B. Oztan is with the Electrical and Computer Engineering Department, University of Rochester, Rochester, NY 14627-0126 USA (e-mail: basak.oztan@rochester.edu).

G. Sharma is with the Electrical and Computer Engineering Department and the Department of Biostatistics and Computational Biology, University of Rochester, Rochester, NY 14627-0126 USA (e-mail: gaurav.sharma@ rochester.edu).

This paper has supplementary PDF material available at http://ieeexplore. ieee.org, provided by the authors. The material is in $92 \mathrm{MB}$ file size. The supplementary material contains the full-scale scan of the self-modulated Library halftone shown in Fig. 21(a) in this paper that allows the self-modulation to be observed more clearly.

Digital Object Identifier 10.1109/TIP.2009.2028367 process [6] in the sense that the halftone spots (clusters of individual printer dots) are centered at a periodic array of locations. Different shades of gray are reproduced by varying the size of these spots. The use of a single halftone cell to tile the image plane minimizes memory requirements and simplifies computation; considerations that were important in the early days of digital halftoning when these resources were rather expensive. These constraints become less stringent in current digital systems and one can explore the flexibility to develop alternative digital halftoning methods that utilize more of these resources but offer improvements in functionality or performance.

In this paper, we describe a method to modulate the phase of digital clustered-dot halftone screens dynamically during the halftoning process. Instead of using a halftone threshold array, we employ an analytic halftone threshold function, whose phase can be modulated independently using an auxiliary signal. Suitable design of the auxiliary signal allows us to address two different applications that we describe subsequently. Since discontinuities in the modulated phase may produce visible artifacts in the halftones, we impose a requirement of continuity on the modulated phase. Using the analogy with continuous phase modulation in digital communications [7, pp. 598-602], we refer to the resulting technique as continuous phase-modulated $(C P M)$ halftoning. By exploiting the relation between frequency and phase, modulation of the screen frequency is also accomplished within the CPM framework, albeit with additional constraints. In order to gain insight into the limitations and capabilities of CPM halftones, we examine their spectral characteristics by using a simplified analytic model.

We consider two practical applications of CPM halftones. First, we demonstrate how the phase (or frequency) modulation may be exploited in order to embed a watermark pattern in the halftone image [1]. Next, we describe how the traditional trade-off between spatial and tonal resolution for clustered-dot halftones can be ameliorated by varying the screen frequencies according to the frequency content of the contone image. We refer to this adaptive halftoning technique as self-modulated halftones [2].

The rest of the paper is organized as follows. Section II reviews existing work on related applications and summarizes our contributions. In Section III, modulating halftone phase/frequency using CPM halftoning is described. Applications exploiting CPM halftones are described in Section IV along with their limitations and the experimental results are presented in Section V. Finally, in Section VI, we present conclusions.

\section{RELATED WORK}

Both of the applications we consider have previously been addressed in the literature. For centuries, conventional paper watermarks have been utilized for security and forensics applications [8, pp.64-68], [9]. In the digital domain, halftone watermarks provide a functionality that mimics or extends the 
capabilities of the conventional paper watermarks [8, Ch.5], [10], [11]. Several watermarking techniques have been previously proposed for digital clustered-dot halftones [12]-[16]. Among these, methods based on modulating the phase or screen frequency have also been investigated. Varying the halftone screen frequency by warping the spatial domain by a sinusoidal waveform was proposed by Ostromoukhov et al. for protection from counterfeiting [17]. In [18], Wang proposed a method to generate clustered-dot halftone screens with clusters centered stochastically on the screen. The method allows phase and frequency variations within the screen and was adopted in [19]-[22] to embed textual watermark patterns in clustered-dot halftones by modulating the phase of the halftone screen according to the watermark pattern. The embedded watermark pattern can be visually detected by using an inexpensive transparency decoder or by scanning the printed image and processing it using a computer [20]. Through suitable design, the watermark may also be decoded without requiring any additional equipment [21]. These watermarking techniques require the generation of a (relatively) large threshold array with the embedded information built into the phase variation over the multiple "halftone cells" within the array. This array typically needs to be designed in advance with the tiling requirements in mind, which limits flexibility because only watermarks for which tiles have been predesigned can be embedded. CPM halftones, however, allow the information to be dynamically embedded during the halftoning process and, thus, offer a simpler and more flexible solution. As with other methods, the embedded information can be retrieved using either a simple scan-shift-overlay process or a reference transparency overlay.

The spatial and tonal rendering of clustered-dot halftones strongly depend upon trading spatial resolution for tonal resolution [5, pp. 399-400], [23], [24]. The number of gray levels that can be reproduced in a digital clustered-dot halftone depends on the size of the halftone cell that is used to tile the image plane. The number of halftone cells that fit within a linear unit distance in the direction of halftone screen orientation is referred to as the screen frequency, which is commonly measured in lines per inch (lpi). A low screen frequency halftone offers more tonal but less spatial resolution due to the large and coarsely spaced halftone spots. On the other hand, the smaller and closely spaced halftone spots in a high screen frequency halftone allow better rendering of spatial details despite the limited number of gray levels that can be reproduced. A variety of solutions have been proposed to enhance the spatial and tonal rendering of halftones. Though conventional methodologies usually considered these two aspects separately [25]-[27], both of these aspects can be jointly improved. Larger size halftone cells can be used for the rendering of smooth regions such as backgrounds to improve the tonal resolution and smaller size cells can be employed at spatially varying regions such as edges to increase the spatial resolution. This image adaptive approach is adopted in [28] and [29] by varying the size of the dither matrices depending on the changes in image values along the scanning path of a space filling curve. In [30], Hel-Or et al. developed a similar iterative halftoning scheme, wherein the halftone cells vary in size and shape based on a spatial "busyness" metric for the image. These methods tend to be computationally expensive. Self-modulated halftones based on CPM offer a simpler alternative.

Our presentation in this paper also includes an analysis of CPM halftones in the context of these two proposed applications, which has not been addressed in prior work. Both considerations of visibility of the modulation and detectability of embedded watermarks are considered in this analysis.

\section{Continuous Phase-Modulated HalfTONES}

\section{A. Analytic Halftone Generation With Phase Modulation}

A clustered-dot digital halftone image for a monochrome contone image $\mu(x, y)$ is commonly generated by comparing the image values against a periodic halftone threshold function $T(x, y)$, where $x$ and $y$ represent the spatial coordinates along the horizontal and vertical directions, respectively. Specifically

$$
h(x, y)= \begin{cases}1, & \text { if } \mu(x, y)<T(x, y) \\ 0, & \text { otherwise }\end{cases}
$$

defines the halftone image, where the values 1 and 0 correspond to the respective decisions that ink/toner is, or is not, deposited at the pixel position $(x, y)$.

As described in Section I, the halftone threshold function is typically a predesigned periodic array of spatially varying threshold values, whose arrangement depends on the halftone parameters such as screen frequency, orientation, printer addressability, and spot function. Manipulation of the halftone parameters through an adjustment of the threshold array values tends to be an unwieldy process and analytic functional descriptions of the halftones are, therefore, useful when such modifications are desired [31]-[33]. For images assuming intensity values in the interval $[-1,1]$ and orthogonal screens, Pellar and Green [32], [33] defined a useful threshold function as

$$
T(x, y)=\cos \left(2 \pi f_{x} x\right) \cos \left(2 \pi f_{y} y\right)
$$

where $f_{x}$ and $f_{y}$ represent the screen frequencies along the orthogonal $x$ and $y$ axes, respectively.

In order to allow controlled phase variations in the halftone screen, we propose a modification of this function by introducing two spatially varying phase functions $\Psi_{\mathbf{X}}(x, y)$ and $\Psi_{\mathbf{Y}}(x, y)$ in the respective cosine function arguments [1], thereby obtaining a generalized form of the analytic halftone threshold function as

$$
\begin{aligned}
& T(x, y) \\
& \quad=\cos \left(2 \pi f_{x} x+\Psi_{\mathbf{X}}(x, y)\right) \cos \left(2 \pi f_{y} y+\Psi_{\mathbf{Y}}(x, y)\right) .
\end{aligned}
$$

Spatial discontinuities in the modulated phase can cause visible artifacts in the printed halftones. Continuity and smoothness of these signals are required to eliminate/reduce the visibility of these artifacts. Consequently, we refer to the resulting halftoning technique as continuous phase-modulated (CPM) halftoning. This methodology can be exploited to embed signals in the halftone image. Specifically, we describe how a watermark pattern can be embedded by controlling the halftone phase in Section IV-A.

The halftone images in Fig. 1 illustrate the effect of different levels of phase modulation on the halftone appearance. Fig. 1(a) 


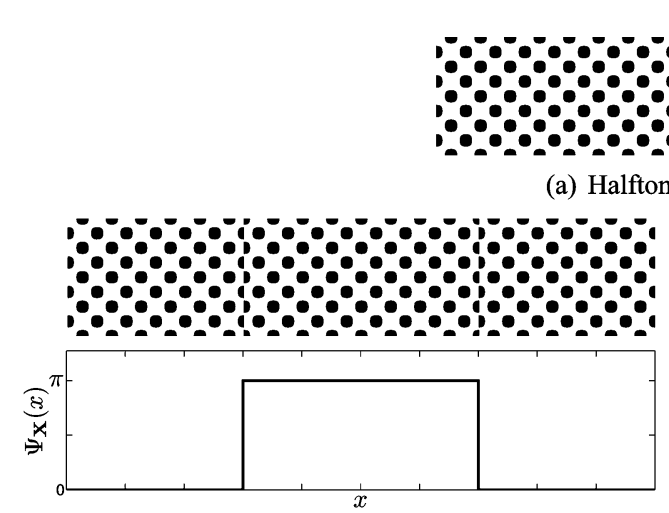

(b) Phase-modulated halftone with visible artifacts

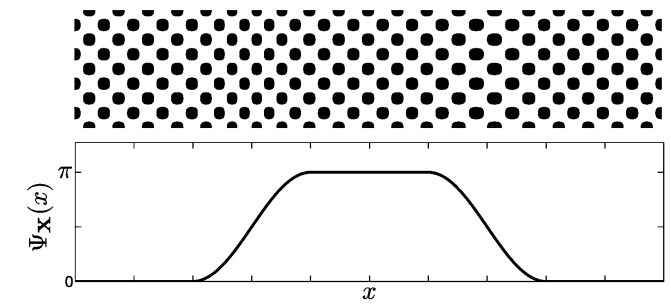

(c) Continuous phase-modulated halftone

Fig. 1. Illustration of phase modulation on the halftone appearance: (a) shows the reference halftone image without any modulation; (b) shows a phase-modulated halftone with visible artifacts induced by the discontinuities in the phase; (c) shows a continuous phase-modulated halftone without any artifacts.

shows the halftone image generated for a constant gray level contone image using the threshold function of (3) without any modulation in its phase. In Fig. 1(b) and (c), the phase of the threshold function is modulated only along the horizontal axis with the signals $\left(\Psi_{\mathbf{X}}(x)\right)$ shown beneath each halftone image. While the sudden rise and fall in the modulated phase yield visible artifacts in the halftone image of Fig. 1(b), CPM provides seamless transitions in the halftone image of Fig. 1(c).

\section{B. Analytic Halftone Generation With Frequency Modulation}

Continuous phase modulation of clustered-dot halftones can also be utilized to vary the screen frequencies in different regions of the printed image. Frequency modulation is accomplished by controlling the rate of change in the instantaneous phase, i.e., the instantaneous frequency, of a carrier signal. Closed form expressions for the instantaneous frequencies of the modified threshold function (3) are hard to obtain. If the phase modulation terms $\Psi_{\mathbf{X}}(x, y)$ and $\Psi_{\mathbf{Y}}(x, y)$ do not vary along the directions orthogonal to their subscripted indices (for instance, $\Psi_{\mathbf{X}}$ does not vary along $y$ ), they can be represented by two 1-D functions $\Psi_{\mathbf{X}}(x)$ and $\Psi_{\mathbf{Y}}(y)$, respectively, and, consequently, (3) becomes a separable function like (2). Under this assumption, the instantaneous frequencies along the horizontal and vertical axes are simply given by 1

$$
\begin{aligned}
& f_{x}^{i}(x)=f_{x}+\frac{1}{2 \pi} \frac{d \Psi_{\mathbf{X}}(x)}{d x} \text { and } \\
& f_{y}^{i}(y)=f_{y}+\frac{1}{2 \pi} \frac{d \Psi_{\mathbf{Y}}(y)}{d y}
\end{aligned}
$$

respectively.

A frequency-modulated sinusoidal carrier signal in the time domain is commonly represented by $\cos \left(2 \pi f_{c} t+2 \pi \beta \int_{-\infty}^{t} \phi(\tau) d \tau\right)$ such that the instantaneous frequency at time $t$ is given by $f_{c}+\beta \phi(t)$ [7, pp. 328-329]. This convention has also been applied to digital

\footnotetext{
${ }^{1}$ Through a rotation of the coordinate axes, method can also readily handle orthogonal halftone screens that are not necessarily oriented along $0 / 90^{\circ}$, such as the common $45^{\circ}$ orientation. For notational simplicity, however, we consider only the "vertical-horizontal" case.
}

modulation [34]. Mimicking conventional FM, if the phase terms are defined as

$$
\begin{aligned}
& \Psi_{\mathbf{X}}(x)=2 \pi \beta_{x} \int_{-\infty}^{x} \Phi_{\mathbf{X}}(\tau) d \tau \text { and } \\
& \Psi_{\mathbf{Y}}(y)=2 \pi \beta_{y} \int_{-\infty}^{y} \Phi_{\mathbf{Y}}(\nu) d \nu
\end{aligned}
$$

then using (4) and (5), the instantaneous frequencies are readily expressed as

$$
\begin{aligned}
& f_{x}^{i}(x)=f_{x}+\beta_{x} \Phi_{\mathbf{X}}(x) \text { and } \\
& f_{y}^{i}(y)=f_{y}+\beta_{y} \Phi_{\mathbf{Y}}(y)
\end{aligned}
$$

where $\beta_{x}$ and $\beta_{y}$ are the frequency deviation constants and $\Phi_{\mathbf{X}}(x)$ and $\Phi_{\mathbf{Y}}(y)$ are the modulating functions for the modulation along the horizontal and vertical directions, respectively. Variations in the screen frequencies are introduced by suitably controlling these parameters. This methodology is exploited for different applications in Sections IV-B and IV-C.

For the purpose of illustration, Fig. 2(a) and (b) show no frequency-modulated halftone images obtained by choosing the frequency deviation constants $\beta_{x}=f_{x}$ and $\beta_{y}=0$, and using the modulating functions $\left(\Phi_{\mathbf{X}}(x)\right)$ as shown beneath each halftone image. Neither of the frequency-modulated halftone images exhibit any objectionable artifacts due to the inherent continuity of the modulated phase, nonetheless, the smoother modulating function in Fig. 2(b) yields a more appealing halftone image.

\section{Spectral Characteristics of Continuous Phase-Modulated Halftones}

The perception of halftones depends on the human visual system (HVS), where the sensitivity of the observer to the variations in the image is affected by the spatial frequency [35]. It is well-known from the communication theory that the modulation in phase or frequency leads to a widening in the signal bandwidth [7, pp. 333-343]. In this context, if the visual system has substantial sensitivity in the regions where the signal spectrum expands, then the modulation can introduce visible distortions in the halftone image. Therefore, understanding the spectral characteristics of the CPM halftones helps assess visibility of the modulated halftone images. 


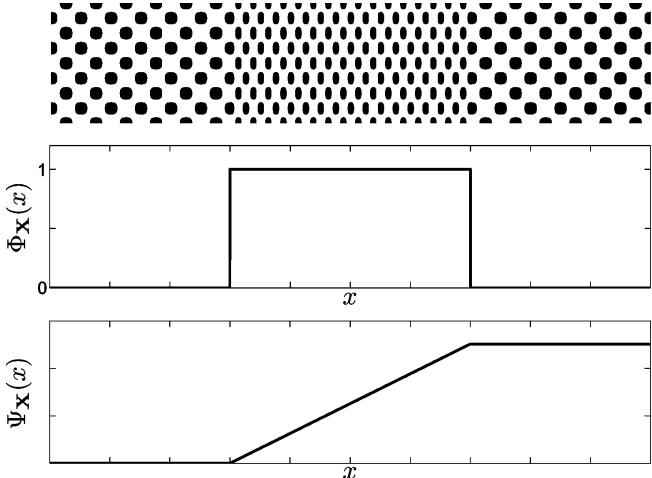

(a) Frequency-modulated halftone with a discontinuous modulating function

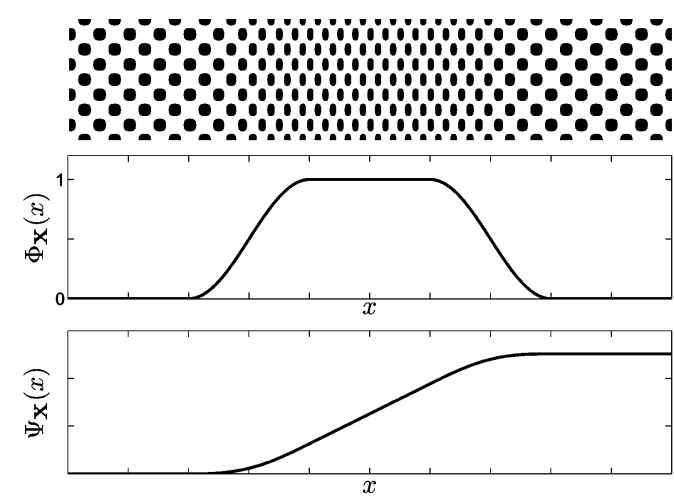

(b) Frequency-modulated halftone with a smoother modulating function

Fig. 2. Illustration of frequency modulation on halftone appearance. Frequency-modulated halftones do not exhibit any discontinuity artifacts since the modulated phase is inherently continuous. Nevertheless, the smoother modulating function shown in (b) results in a more appealing halftone image than the one shown in (a).

Spectral characterization of an arbitrarily modulated halftone in closed form is difficult. We simplify the problem by assuming that the halftone is formed by rectangular halftone spots ${ }^{2}$ and the modulations along the horizontal and vertical directions are independent. Hence, the halftone can be modeled as the product of two 1-D modulated pulse sequences along each of the orthogonal axes and we focus on estimating the spectral characteristics of a 1-D modulated pulse sequence, which provides useful insight for the 2-D scenario. From the digital modulation perspective, a phase-modulated pulse sequence can be considered as a pulse-position modulated signal, while a frequency-modulated pulse sequence can be modeled by a pulse-frequency modulated waveform. Under some constraints on the statistics of the modulating signal, the power spectral density of the pulse-modulated signal can be estimated [36]. The smoothness and continuity requirement of the modulating waveform, however, motivates consideration of a simpler deterministic signal with these properties. Therefore, we assume periodic modulation, specifically with a sinusoid, in phase or frequency, and estimate the spectra of the modulated halftones (pulse sequences).

We first consider a phase-modulated halftone with a normalized average gray level $\tau_{p} / \tau$ in the form of a pulse-position modulated waveform as illustrated in Fig. 3. The halftone is comprised of spots of width $\tau_{p}$ each of which is located within a halftone cell that has width $\tau$. The modulation in phase is modeled as a shift of the halftone "pulse" within the cell, yielding an overall model for the phase-modulated halftone as

$$
h_{P M}(x)=\sum_{n=-\infty}^{\infty} s\left(x-n \tau-\varepsilon_{n}\right)
$$

where the $s(x)$ is a rectangular pulse of width $\tau_{p}$, symmetrically centered in the interval $[0, \tau]$, and $\varepsilon_{n} \in\left[-\tau_{d}, \tau_{d}\right]$ is the shift corresponding to the $n$th halftone cell. Consider a sinusoidally varying modulation signal $\varepsilon_{n}=\tau_{d} \sin (2 \pi n / N+\phi)$, where $\phi \in[-\pi, \pi]$ is the initial phase, and $N$ is a positive integer representing the period of $\varepsilon_{n}$ in number of halftone cells. It is

\footnotetext{
${ }^{2}$ The analysis can be generalized to the 2-D case with circular halftone spots in a conceptually straightforward fashion, albeit at the expense of significant notational complexity.
}

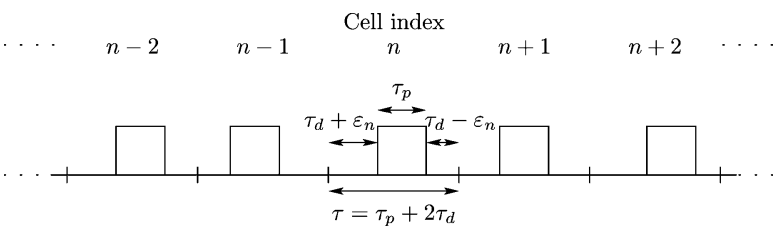

Fig. 3. Pulse-position modulated halftone.

clear that $h_{P M}(x)$ is periodic with period $N \tau$ and, thus, it can be represented in terms of a Fourier series as

$$
\begin{aligned}
& h_{P M}(x)=a_{0_{P M}} \\
& \quad+2 \sum_{k=1}^{\infty}\left[a_{k_{P M}} \cos \left(\frac{2 \pi k}{N \tau} x\right)+b_{k_{P M}} \sin \left(\frac{2 \pi k}{N \tau} x\right)\right]
\end{aligned}
$$

where (see details in Appendix A) $a_{0_{P M}}=\tau_{p} / \tau$ and the remaining Fourier series coefficients are given by

$$
\begin{aligned}
a_{k_{P M}}= & \frac{\tau_{p}}{N \tau} \operatorname{sinc}\left(\frac{k \tau_{p}}{N \tau}\right) \\
& \sum_{n=0}^{N-1} \cos \left(\frac{2 \pi k}{N \tau}\left(n \tau+\varepsilon_{n}+\frac{\tau}{2}\right)\right) \\
b_{k_{P M}}= & \frac{\tau_{p}}{N \tau} \operatorname{sinc}\left(\frac{k \tau_{p}}{N \tau}\right) \\
& \sum_{n=0}^{N-1} \sin \left(\frac{2 \pi k}{N \tau}\left(n \tau+\varepsilon_{n}+\frac{\tau}{2}\right)\right)
\end{aligned}
$$

for $k=1,2, \ldots, \infty$.

Next, we consider a frequency-modulated halftone in the form of a pulse-frequency modulated waveform, where the modulation in frequency does not change the normalized average gray level $\tau_{p} / \tau$ in a given halftone cell. Motivated by this constraint, a hybrid pulse-frequency-width modulated sequence is illustrated in Fig. 4, which can be mathematically expressed as

$$
h_{F M}(x)=\sum_{n=-\infty}^{\infty} s_{n}\left(x-\sum_{m=-\infty}^{n-1} \tau_{m}\right)
$$




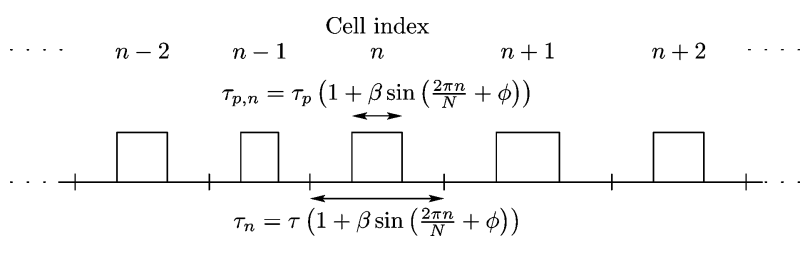

Fig. 4. Hybrid pulse-frequency-width modulated halftone.

where $s_{n}(x)$ is a rectangular pulse of width $\tau_{p, n}$ symmetrically centered in the interval $\left[0, \tau_{n}\right]$. The sinusoidally modulated width and period of the pulse are assumed to be

$$
\begin{aligned}
\tau_{p, n} & =\tau_{p}\left(1+\alpha \sin \left(\frac{2 \pi n}{N}+\phi\right)\right) \text { and } \\
\tau_{n} & =\tau\left(1+\alpha \sin \left(\frac{2 \pi n}{N}+\phi\right)\right)
\end{aligned}
$$

respectively, $\alpha \in[-1,1]$ determines the range of variation in the period $\left(\tau_{n}\right)$, and $N$ is a positive integer representing the period of the modulating signal in number of halftone cells. It can be shown that $h_{F M}(x)$ is a periodic waveform with period $N \tau$, and it can also be represented in terms of a Fourier series as (see details in Appendix B)

$$
\begin{aligned}
& h_{F M}(x)=a_{0_{F M}} \\
& \quad+2 \sum_{k=1}^{\infty}\left[a_{k_{F M}} \cos \left(\frac{2 \pi k}{N \tau} x\right)+b_{k_{F M}} \sin \left(\frac{2 \pi k}{N \tau} x\right)\right]
\end{aligned}
$$

where $a_{0_{F M}}=\tau_{p} / \tau$ and the remaining Fourier series coefficients for $k=1,2, \ldots, \infty$ are

$$
\begin{aligned}
a_{k_{F M}}= & \sum_{n=0}^{N-1} \frac{\tau_{p, n}}{N \tau} \operatorname{sinc}\left(\frac{k \tau_{p, n}}{N \tau}\right) \\
& \cos \left(\frac{2 \pi k}{N \tau}\left(\sum_{m=0}^{n-1} \tau_{m}+\frac{\tau_{n}}{2}\right)\right) \\
b_{k_{F M}}= & \sum_{n=0}^{N-1} \frac{\tau_{p, n}}{N \tau} \operatorname{sinc}\left(\frac{k \tau_{p, n}}{N \tau}\right) \\
& \sin \left(\frac{2 \pi k}{N \tau}\left(\sum_{m=0}^{n-1} \tau_{m}+\frac{\tau_{n}}{2}\right)\right) .
\end{aligned}
$$

The distortion induced by the modulations in phase/frequency can be estimated, to first-order, by observing the change in the total visible power of the halftone. Visual system models commonly employ a linear shift-invariant filter to approximate the contrast sensitivity function (CSF) of an average observer as a function of the spatial frequency. Representing the magnitude frequency response of the visual system by $C S F(f)$ and using the Fourier series representation of the modulated halftones, the total visible power of the modulated halftones is obtained as

$$
P_{\text {visible }}=\left(C S F(0) a_{0}\right)^{2}+2 \sum_{k=1}^{\infty} C S F^{2}\left(\frac{k}{N \tau}\right)\left(a_{k}^{2}+b_{k}^{2}\right)
$$

where $a_{0}, a_{k}$, and $b_{k}$ correspond the Fourier series coefficients for the phase-modulated and frequency-modulated halftones.

Even though the HVS is characterized by a band-pass response, for halftoning, it is known that low-pass representations of the HVS are typically better than the band-pass alternatives [37]. We consider a low-pass approximation [38] to the well-known Mannos-Sakrison CSF [39] as (17), shown at the bottom of the page, where $f$ denotes the radial frequency in cycles per degree. The radial frequency in cycles per degree is related to the linear frequency in cycles per inch (cpi) in terms of the viewing distance ${ }^{3} D$, which is assumed to be 12 inches throughout this paper. This function is obtained by setting the CSF to its peak value for all frequencies lower than the frequency at which the peak is attained and then normalizing the "DC-response" (i.e., response at zero-frequency) to unity.

We first investigate the effect of phase modulation on the total visible power. For a set of halftone frequencies, by averaging over several values of the initial phase of the modulating signal $(\phi)$, quantitative estimates of total visible power are obtained as a function of the period of the modulating signal $N$ at 25,50 , and $75 \%$ halftone area coverages and plotted in Fig. 5. It is readily seen that as the period of the modulating signal increases, the variation in phase becomes smoother and the amount of visible degradation it causes decreases. The total visible power of the modulated halftone asymptotically approaches the total visible power of the nonmodulated halftone as $N$ is further increased. Based on this observation, for each of the area coverage and halftone frequency, we empirically choose the smallest period of the modulating signal, equivalently the maximum rate of change in the modulating signal, that ensures the increase in the total visible power does not exceed $10 \%$ of the maximum possible increase. These values are indicated with "cross-marks" in each of the plot in Fig. 5.

The effect of frequency modulation on the total visible power is investigated with a similar approach. The same set of screen frequencies are modulated such that the screen frequency sinusoidally deviates between $\left(f_{x}-\beta_{x}, f_{x}\right)$ cpi with periods that are marked with the cross-marks in Fig. 5 for different area coverages and screen frequencies. The parameter $\alpha$ is determined by the maximum frequency deviation $\beta_{x}$, and total visible power corresponding to the previously used area coverages are estimated as a function of $\beta_{x}$ and shown in Fig. 6. These plots indicate that the modulation in the screen frequency does

${ }^{3} 1 \mathrm{cycles} /$ degree $=\pi D / 180 \mathrm{cycles} /$ inch, where $D$ is the viewing distance in inches.

$$
C S F(f)= \begin{cases}1, & \text { for } f \leq 7.9 \\ \frac{2.6}{0.981}(0.0192+0.114 f) e^{-(0.114 f)^{1.1}}, & \text { otherwise }\end{cases}
$$




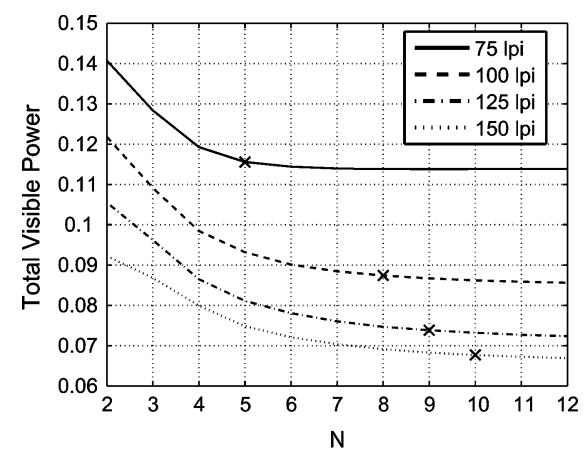

(a) $25 \%$ gray level

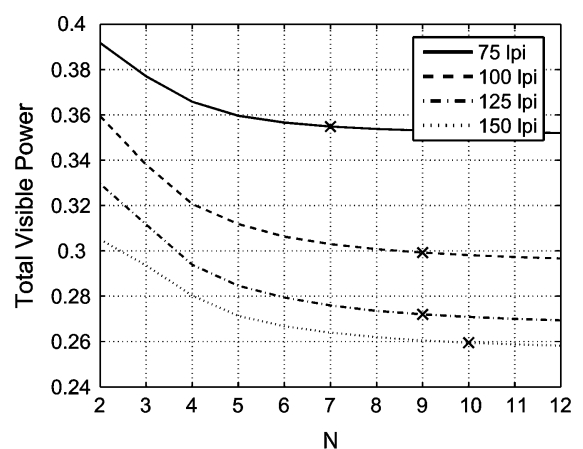

(b) $50 \%$ gray level

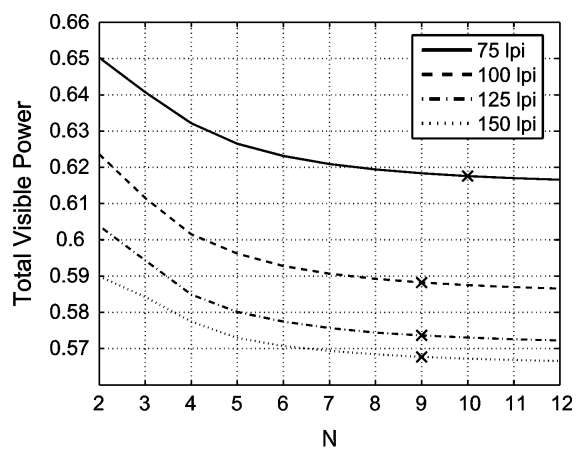

(c) $75 \%$ gray level

Fig. 5. Change in the total visible power of a phase-modulated signal as a function of the period of the modulating signal.

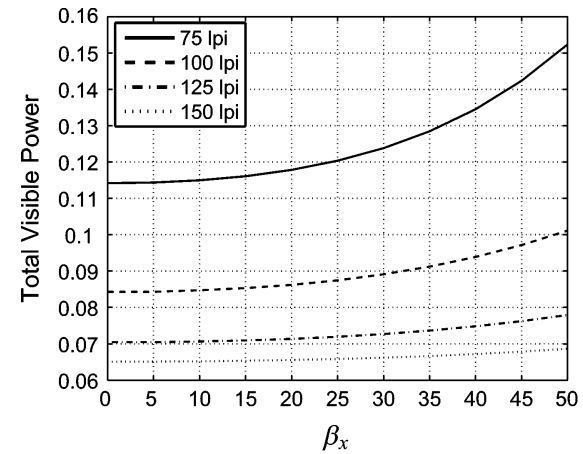

(a) $25 \%$ gray level

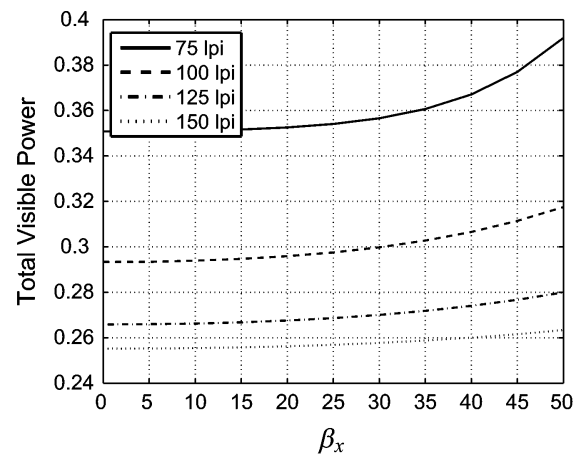

(b) $50 \%$ gray level

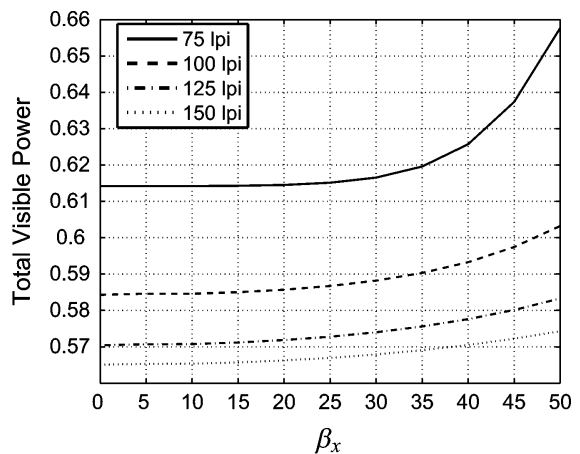

(c) $75 \%$ gray level

Fig. 6. Change in the total visible power of a frequency-modulated signal as a function of the frequency deviation constant.

not increase the visible halftone power significantly even in the scenarios with relatively large frequency modulation constants. However, modulations can still be perceptible due to the change in the contrast of the modulated halftone texture as we describe in Section IV-C2.

\section{APPLICATIONS}

\section{A. Halftone Watermarking Using Phase Modulation}

Continuous phase modulation can be used to embed watermarks in halftones. In this section, we describe how a watermark pattern can be embedded in the halftone by controlling the variations in phase. While the embedded functions may be arbitrary subject to the continuity requirement, we consider here the embedding of visual patterns similar to those used in [19]-[22]. A watermark pattern is used to generate a 2-D phase map and embedded in the halftone threshold function through continuous phase modulation. Specifically, we embed the watermark by using one of the phase modulation terms in (3) and keeping the other phase term constant throughout the whole image. An example of such a pattern is a bi-level text image in which the sharp edges can be smoothed by a spatial blur to ensure the continuity and smoothness of the phase. The resulting values are then normalized between $[0, \kappa], \kappa \in(0, \pi]$ to introduce a maximum phase change of $\kappa$ radians between the two levels of the watermark pattern, and the halftone image is obtained from the phase-modulated halftone threshold function.

A useful technique to detect the embedded watermark is to overlay the printed image with a transparency bearing a constant gray level (nonmodulated) halftone image that has the same screen frequency as the watermarked halftone image. The average reflectance of the modulated and nonmodulated regions in the watermarked image differ in the resulting overlay, thereby enabling visual detection. In addition to experimental validation of this "demodulation method" [19]-[21], the relationship between the visible pattern and the embedded watermark has also been shown analytically without considering the continuity of the phase and the alignment requirements [22]. A digital simulation of the transparency mask overlay based detection is demonstrated in Fig. 7, where Fig. 7(d) shows the overlay of the continuous phase-modulated halftone in Fig. 7(c) obtained by using the phase modulation term in Fig. 7(a) with the $d e$ coder mask in Fig. 7(b) representing the nonmodulated halftone printed on a transparency. The regions with the modified phase appear darker in the overlay.

An elegant alternative to the transparency mask overlay based detection is to design the watermark pattern in a manner that allows detection using the scanned image alone [20]. An overview of such a method is presented in Fig. 8. The image is partitioned into two regions defined by alternating stripes, each of the stripes being large enough to carry the watermark pattern to be embedded. The modulating function is then generated as in Fig. 8(a) while the continuity of the phase is ensured along the stripe boundaries. After the CPM halftone is printed and scanned, the image is cyclicly shifted in the vertical direction by one (or more generally an odd number of) stripe height as in Fig. 8(b) such that a stripe with a watermark pattern is paired with a one with no marking. In this way, a stripe with no marking 


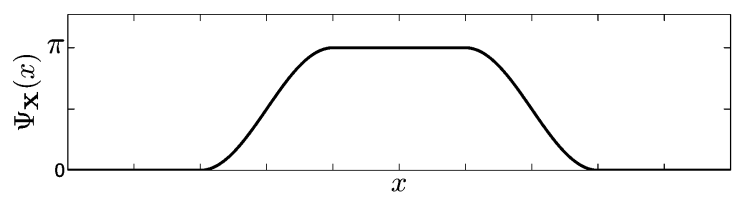

(a) Phase modulation term

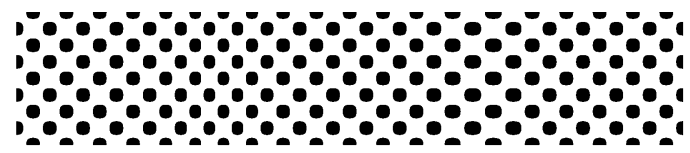

(c) Phase-modulated halftone

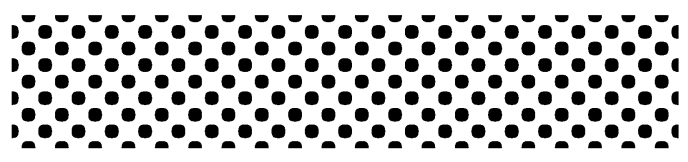

(b) Decoder mask

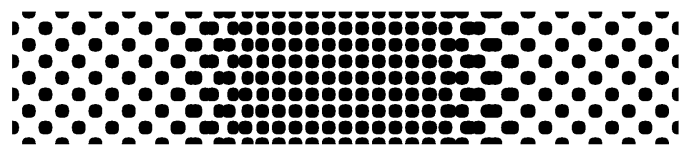

(d) Overlay of the phase-modulated halftone with the decoder mask

Fig. 7. Overlay of phase-modulated halftone with the decoder mask.

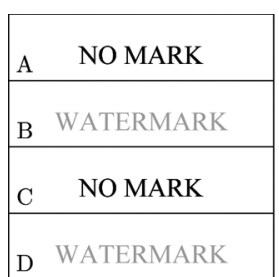

(a)

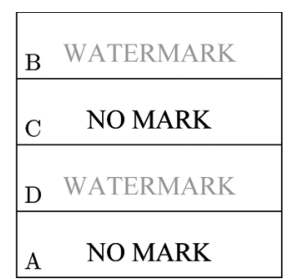

(b)

\begin{tabular}{||c|}
\hline WATERMARK \\
\hline WATERMARK \\
\hline WATERMARK \\
\hline WATERMARK \\
\hline
\end{tabular}

(c)
Fig. 8. Overview of self-referencing detection method. (a) shows the spatial distribution of watermarks in modulating function and correspondingly in scanned image, (b) shows the image after the cyclic-shift of odd number of stripes in the scanned image, and (c) shows the striped pattern of repeated watermark patterns visible in the resulting overlay.

mimics the decoder mask that is used in the transparency overlay based detection method. The images are then digitally superimposed and the watermark patterns become visible in the resulting overlay as shown in Fig. 8(c). This scanned image based detection method is referred to as a self-referencing detector.

1) Detectability of the Embedded Watermark Pattern: Phase modulation enables visual detection by inducing variations in the overlay reflectance. Characterization of the overlay reflectance as a function of the embedded watermark pattern, reflectances of the constituent halftones, and the alignment between the constituent halftone images is, therefore, useful in order to understand the visual detection process. We consider a 2-D halftone $h_{1}(x, y)$ with average reflectance $1-\mu_{1}$ obtained by using the constant phase modulation terms $\Psi_{\mathbf{X}}(x, y)=\psi_{x}$ and $\Psi_{\mathbf{Y}}(x, y)=\psi_{y}$ in the threshold function (3). By exploiting the relation between phase and spatial displacement, we model these constant phase shifts as constant spatial displacements of the halftones spots by $d_{x}$ and $d_{y}$ in halftone cell units along the horizontal and vertical directions, respectively, where $d_{x}=\psi_{x} / 2 \pi$ and $d_{y}=\psi_{y} / 2 \pi$. In order to detect the modulations in phase, $h_{1}(x, y)$ is superimposed with a nonmodulated halftone of the same screen frequency $h_{2}(x, y)$ with average reflectance $1-\mu_{2}$. The average reflectance of the resultant overlay $h_{o v}(x, y)$ as a function of the halftone area coverages $\mu_{1}, \mu_{2}$, and the displacements $d_{x}, d_{y}$ can be obtained as

$$
\begin{aligned}
R_{o v}\left(\mu_{1}, \mu_{2}, d_{x}, d_{y}\right)= & 1-\mu_{o v}\left(\mu_{1}, \mu_{2}, d_{x}, d_{y}\right) \\
\mu_{o v}\left(\mu_{1}, \mu_{2}, d_{x}, d_{y}\right)= & \mu_{1}+\mu_{2}-\sum_{n, m=-1}^{1} \int_{-0.5}^{0.5} \int_{-0.5}^{0.5} \\
& s_{\mu_{1}}\left(x-d_{x}, y-d_{y}\right) \\
& \times s_{\mu_{2}}(x-n, y-m) d y d x
\end{aligned}
$$

where $s_{\mu_{1}}(x, y)$ and $s_{\mu_{2}}(x, y)$ are the halftone spots for $h_{1}(x, y)$ and $h_{2}(x, y)$, respectively. Though quantitative estimates can be obtained via suitable models for rather general 2-D halftone geometries [40], significant insight can be gained by using a 1-D simplification on this model as shown in Fig. 9(a) that allows us to obtain analytical expressions that characterize the visual detection process. The 1-D halftone $h_{1}(x)$ with average reflectance $1-\mu_{1}=1-\tau_{1} / \tau$, where $\tau_{1}$ and $\tau$ are the width of the halftone spot and cell, is phase-modulated using the constant phase term $\Psi_{\mathbf{X}}(x)=\psi$. The spatial displacement corresponding to this phase shift is represented by $d$, where $d=$ $\psi / 2 \pi$ in halftone cell units. The resultant overlay $h_{o v}(x)$ is formed by superposing $h_{1}(x)$ with the nonmodulated halftone of the same screen frequency $h_{2}(x)$ with average reflectance $1-\mu_{2}$ to detect the change in halftone phase. The average reflectance of $h_{o v}(x)$ as a function of the halftone area coverages $\mu_{1}, \mu_{2}$, and the displacement $d$ can be expressed as ${ }^{4}$ (19), shown at the bottom of the page, where

$$
\left(\frac{\mu_{1}+\mu_{2}}{2}\right)_{e}= \begin{cases}\frac{\mu_{1}+\mu_{2}}{2}, & \text { if } \frac{\mu_{1}+\mu_{2}}{2} \leq 0.5 \\ 1-\frac{\mu_{1}+\mu_{2}}{2}, & \text { otherwise. }\end{cases}
$$

${ }^{4}$ Equation (19) can be readily obtained for the case of $\mu_{1}, \mu_{2} \leq 0.5$ and generalized for other cases through mirror symmetry.

$$
\begin{aligned}
& R_{o v}\left(\mu_{1}, \mu_{2}, d\right)=1-\mu_{o v}\left(\mu_{1}, \mu_{2}, d\right) \\
& \mu_{o v}\left(\mu_{1}, \mu_{2}, d\right)= \begin{cases}\max \left(\mu_{1}, \mu_{2}\right), & \text { if }|d| \leq\left|\frac{\mu_{1}-\mu_{2}}{2}\right| \\
|d|-\left|\frac{\mu_{1}-\mu_{2}}{2}\right|+\max \left(\mu_{1}, \mu_{2}\right), & \text { if }\left|\frac{\mu_{1}-\mu_{2}}{2}\right| \leq|d| \leq\left(\frac{\mu_{1}+\mu_{2}}{2}\right)_{e} \\
\min \left(\mu_{1}+\mu_{2}, 1\right), & \text { if }\left(\frac{\mu_{1}+\mu_{2}}{2}\right)_{e} \leq|d| \leq 0.5\end{cases}
\end{aligned}
$$




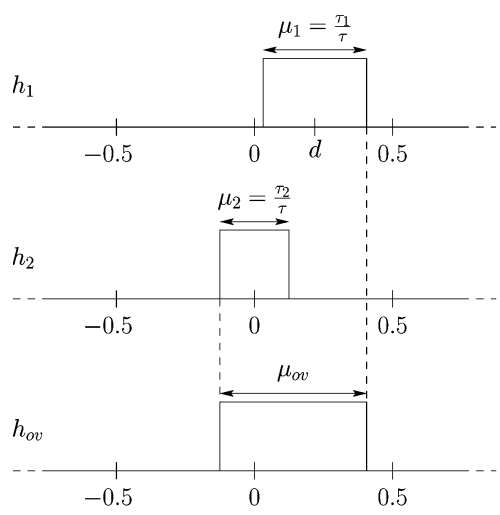

(a)

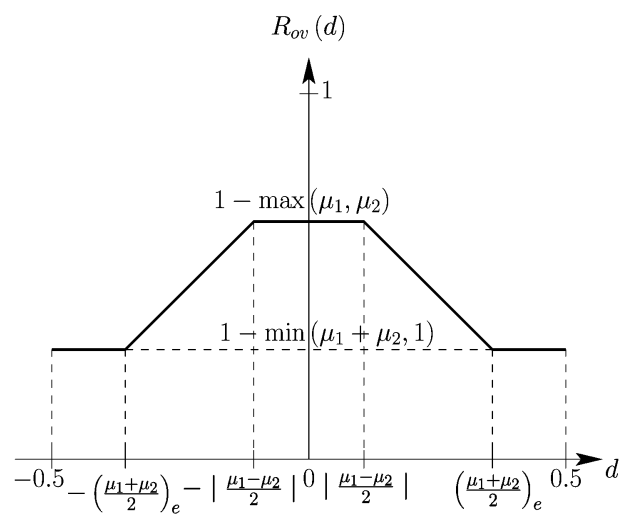

(b)

Fig. 9. Analysis model for computing the average reflectance of the halftone overlay: (a) shows the halftone cells that include the origin; (b) shows the average reflectance versus the spatial displacement $d$ for an arbitrary $\mu_{1}, \mu_{2}$ pair.

Complete graphical representation of $R_{o v}\left(\mu_{1}, \mu_{2}, d\right)$ is infeasible due to the large number of variables. We, therefore, illustrate a few specific cases of interest. First, we consider a generic case for an arbitrary $\mu_{1}, \mu_{2}$ pair and plot $R_{o v}(d)$ in Fig. 9(b). $R_{o v}(d)$ has a linear (strictly affine) dependence on $d$ over the two intervals defined by $\left|\left(\mu_{1}-\mu_{2}\right) / 2\right| \leq|d| \leq$ $\left(\left(\mu_{1}+\mu_{2}\right) / 2\right)_{e}$, and is invariant to the displacement $d$ over the remaining range of displacements (because the two halftone spots have maximum/minimum overlap).

Next, we investigate the maximum possible change in the average reflectance induced by the modulation in phase as a function of individual halftone area coverages $\mu_{1}$ and $\mu_{2}$. This analysis particularly determines the gray levels that are more suitable for embedding the watermark pattern and also determines an optimal range of area coverages for the decoder mask. From (19), one can readily see that the maximum possible change in the average reflectance is $\min \left(\mu_{1}+\mu_{2}, 1\right)-\max \left(\mu_{1}, \mu_{2}\right)$. This relation is illustrated in the perspective 3-D plot in Fig. 10. Zero $\left(\mu_{1}=0\right.$ or $\left.\mu_{2}=0\right)$ and one-hundred $\left(\mu_{1}=1\right.$ or $\left.\mu_{2}=1\right)$ percent halftone area coverages represent the trivial cases that do not allow any change in the overlay reflectance due to the absence of halftone structure at these area coverages. In addition, highlight and shadow gray levels also offer a limited contrast for the recovered watermark and should, therefore, be avoided for the decoder mask. Midtone gray levels allow larger changes in the contrast for the recovered watermark and, therefore, should be favored for the decoder mask. In fact, the $50 \%$ area coverage yields the largest change in the overlay reflectance and serves as the ideal area coverage parameter for the decoder mask.

Our analysis can now be utilized to explore the watermark detectability for the transparency mask overlay and self-referencing based detection algorithms. In both cases we consider the change in overlay reflectance for a constant phase modulation $\Psi_{\mathbf{X}}(x)=\psi$, characterized by the corresponding displacement $d$. As indicated in the preceding paragraph, for the transparency mask overlay based detection method a $50 \%$ area coverage halftone serves as an ideal decoder mask. For this case, the reflectance $R_{o v}^{r}(\mu, d)$ of the overlay produced in the detection process is obtained from (19) as $R_{o v}^{r}(\mu, d)=R_{o v}(\mu, 0.5, d)$, where $\mu$ denotes the area coverage of the (cover) image halftone and $d$ the phase modulation. For the self-referencing detection

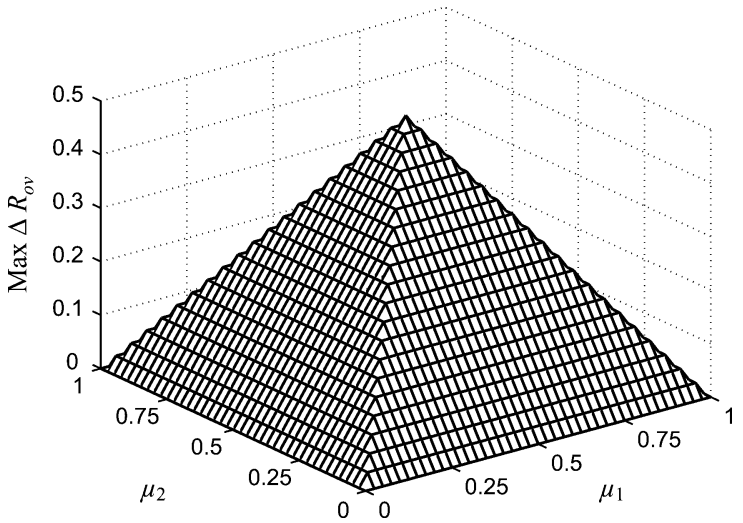

Fig. 10. Maximum amount of change in reflectance of the overlay as a function of $\mu_{1}$ and $\mu_{2}$.

method, an adjoining stripe serves as a reference for each stripe during the detection process. Thus, appealing to the smoothness of typical images, we can approximate the detection process as one in which the overlaid reference transparency has the same halftone area coverage as the (cover) image halftone. Using this approximation, $R_{o v}^{s}(\mu, d)=R_{o v}(\mu, \mu, d)$ denotes the reflectance of the overlay produced in the detection process in terms of the parameters $\mu$ and $d$ defined as before. The reflectance $R_{o v}^{r}(\mu, d)$ and $R_{o v}^{s}(\mu, d)$ are plotted in Fig. 11(a) and (b), respectively. As indicated earlier, these represent the observed reflectance in the overlay produced in the watermark detection for the decoder mask based and self-referencing detection methods, respectively. Using these plots and the corresponding mathematical expressions, one can also determine the spatial variations in reflectance observed in the overlay created in the detection process for a slowly varying modulating patter $d(x)$ as a function of the halftone image area coverage $\mu$ (or equivalently the image gray level). Specifically, for the decoder mask based detection, the reflectance observed in the overlay is obtained as $R_{o v}^{r, \mu}(x)=R_{o v}^{r}(\mu, d(x))$ and the corresponding expression for the self-referencing detection is $R_{o v}^{s, \mu}(x)=R_{o v}^{s}(\mu, d(x))$.

Several characteristics of the detection process become immediately apparent from these plots. First, as can also be seen 


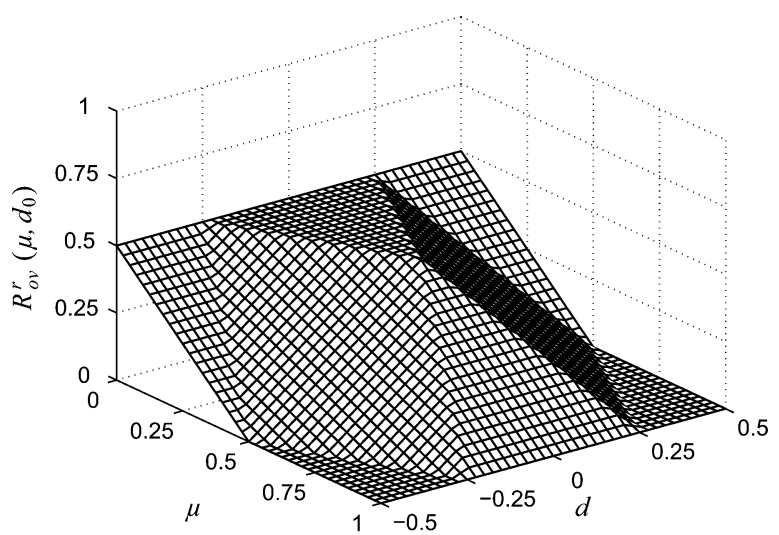

(a) $50 \%$ transparency mask overlay based detector

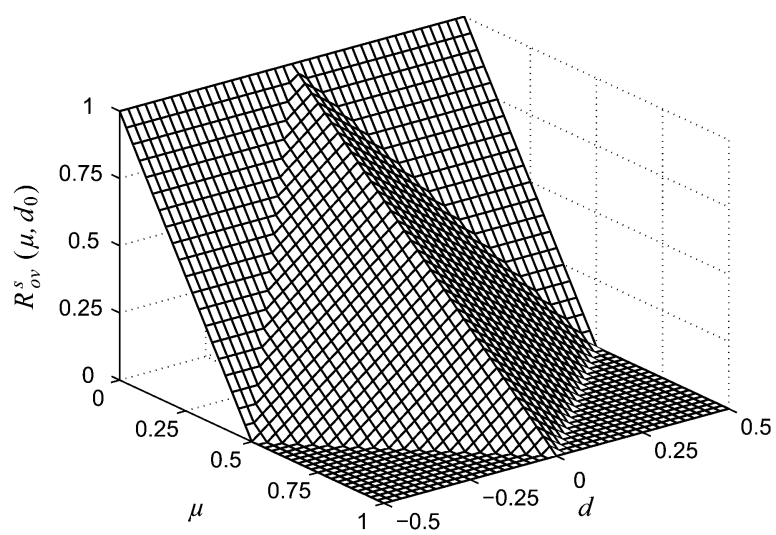

(b) Self-referencing detector

Fig. 11. Overlay reflectance for transparency mask overlay and self-referencing based detection method with respect to halftone area coverage $\mu$ and the displacement $d$.

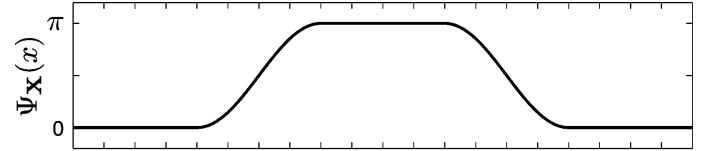

(a) Phase modulation signal

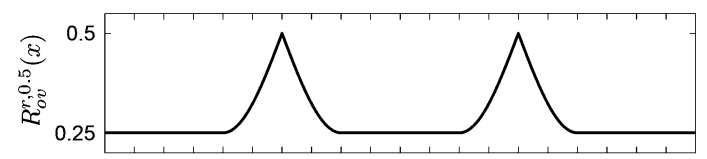

(c) $d_{0}=0.25$

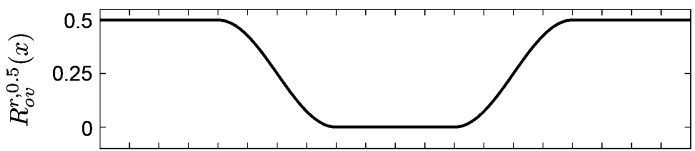

(b) $d_{0}=0$

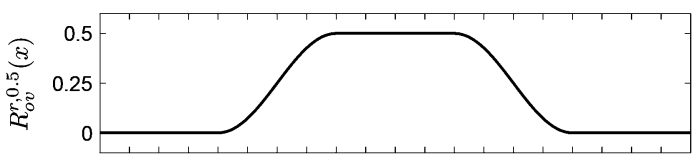

(d) $d_{0}=0.5$

Fig. 12. Example showing the spatial dependence of the overlay reflectance on the modulating signal and the initial misregistration: (a) shows the phase modulation signal; (b), (c), and (d) show demodulated overlay reflectances for misregistration displacements $d_{0}=0,0.25$, and 0.5 , respectively.

from (19), in both these cases the reflectance of the overlay depends only on the magnitude of $d$. As a consequence only the magnitude of the phase modulation can be detected and the sign of the modulation is indistinguishable for these detection methods. Secondly, we can see from Fig. 11(a) and (b) that in both cases, except for a cover image halftone coverage of $50 \%$ $(\mu=0.5)$, there are "dead-zones" over which changes in the modulation do produces no change in the output reflectance. Third, one can see that for the transparency mask based detection the appearance of the overlay obtained in the decoding process depends on the alignment in the overlay. Writing $d(x)=\Psi_{\mathbf{X}}(x) / 2 \pi+d_{0}$, where $-0.5 \leq d_{0} \leq 0.5$ represents the initial displacement between the halftones of the image (in halftone cell width units) and of the decoder mask, we can see that the reflectance patterns observed for the overlay generated in the detection process depend on the initial misregistration $d_{0}$. In particular, the same phase modulation $\Psi_{\mathbf{X}}(x)$ can produce very different appearances based on the misregistration amount $d_{0}$. A specific example of this is shown in Fig. 12. The halftone image in this case was assumed to have an area coverage of $50 \%(\mu=0.5)$. Fig. 12(a) shows the spatial phase modulation pattern $\Psi_{\mathbf{X}}(x)$ and Fig. 12(b)-(d) depict the observed reflectance for this overlay for $d_{0}=0,0.25$, and 0.5 , respectively. Note that in this case one can recover either (a scaled version of) the modulation pattern [Fig. 12(d)], an inverted version [Fig. 12(b)], and a version in which the overlay has a constant region except in the boundary regions so that only the transitions would be seen [Fig. 12(c)]. This dependence on registration has also been previously observed and remarked upon in [21]. Note that the self-referencing detection method does not encounter this ambiguity. By comparing Fig. 11(a) and (b), we can also see that while small displacements may not cause a change in the overlay reflectance in the transparency mask overlay based detection, such situations are not encountered in self-referencing based detection. Therefore, in transparency mask overlay based detection larger amounts of modulation is required to induce a change in the overlay reflectance. If a decrease of $10 \%$ in the overlay reflectance is sufficient to distinguish the modulation in phase, using (19), it can be shown that the minimum amount of modulation in phase to satisfy this constraint is $\pm \pi[|\mu-0.5|+0.2(1-\max (\mu, 0.5))]$ and $\pm 0.2 \pi(1-\mu)$ radians for the $50 \%$ area coverage transparency mask overlay and self-referencing based detection methods, respectively.

\section{B. Halftone Watermarking Using Frequency Modulation}

Continuous phase-modulated halftones can also be exploited for frequency modulation (FM) based watermarking using the framework described in Section III-B. As noted earlier [see (6) and (7)] in this scenario, the modulation needs to be performed in a separable manner. Therefore, in contrast with the phase modulation based embedding of Section IV-A, where the watermark can be defined as a 2-D pattern, for the FM based method one can only embed two independent 1-D patterns through the 


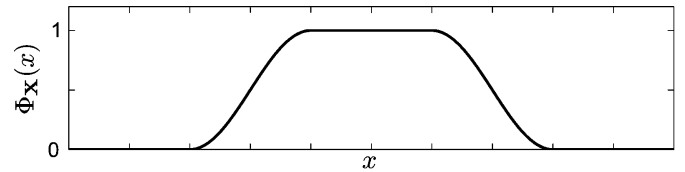

(a) Frequency modulating function

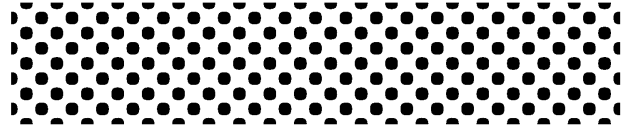

(b) Decoder mask

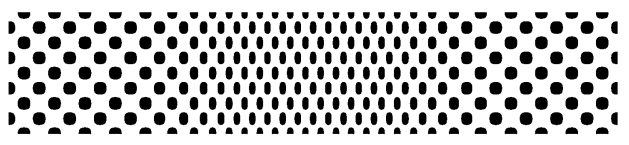

(c) Frequency-modulated halftone

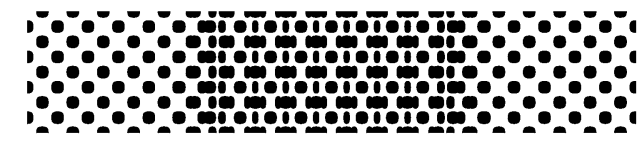

(d) Overlay of the frequency-modulated halftone with the decoder mask

Fig. 13. Overlay of frequency-modulated halftone with the decoder mask.

functions $\Phi_{\mathbf{X}}(x)$ and $\Phi_{\mathbf{Y}}(y)$ in (8) and (9), respectively. While the embedded patterns may be arbitrary, for convenience, we consider embedding binary pulse patterns that introduce a maximum frequency change of $\beta_{x}$ and $\beta_{y}$ cpi, respectively, along the horizontal and vertical directions. From these functions, the corresponding phase terms for CPM are obtained by (6) and (7), which provide the frequency-modulated CPM threshold function (3) from which the halftone is obtained via (1).

The embedded patterns are detected with a technique similar to the detection of the watermark patterns embedded via phase modulation. When the printed image is overlaid with a halftone image of constant screen frequency printed on a transparency, additional low spatial frequency patterns commonly referred to as "moiré" become visible [41]. Suitable choice of the screen frequency of the decoder mask allows distinctive visible moiré frequencies for the different levels of the watermark pattern, thereby allowing visual detection. For the purpose of illustration, the superposition of the frequency-modulated halftone in Fig. 13(c) obtained by using the frequency modulating function in Fig. 13(a) with the decoder mask in Fig. 13(b) is shown in Fig. 13(d), where the frequency-modulated halftone is obtained using CPM with $\beta_{x}=f_{x}$. In the overlay, the regions with the modified frequency exhibit a moiré pattern that clearly highlights the pattern of modulation.

The separability requirement for the halftone threshold function imposes reduced embedding rates. For instance, if $M_{x}$ and $M_{y}$ data bits are embedded along horizontal and vertical directions, respectively, a total of $M_{x}+M_{y}$ data bits can be embedded with separable configurations as opposed to $M_{x} \times M_{y}$ data bits with nonseparable configurations. Nevertheless, the limited capacity may still be useful for specific applications such as ownership verification or meta-data embedding.

1) Detectability of the Embedded Watermark Pattern: The detection of the embedded patterns depends on identifying the moiré patterns in the resultant decoder mask overlaid image. The intensity of the moiré depends on the halftone area coverages of the image and the decoder mask. By using a 1-D model, we obtain analytical expressions for the intensity of the moire that characterize the detectability of the embedded watermark. Let $h_{1}(x)$ and $h_{2}(x)$ represent the image halftone and the decoder mask with screen frequencies $f_{x_{1}}$ and $f_{x_{2}}$, and halftone area coverages $\mu_{1}$ and $\mu_{2}$, respectively. Assume there exist a spatial alignment error $d_{0}$ represents the initial misregistration (in halftone cell units) between the image

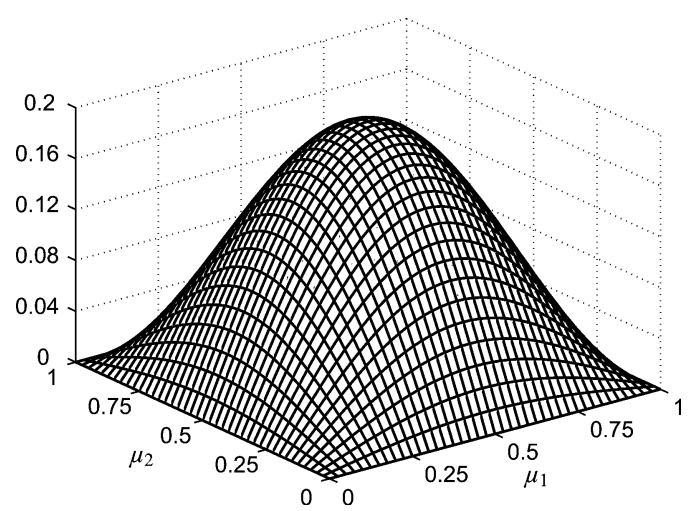

Fig. 14. Amplitude of the $(1,1)$ moiré pattern at frequency $f_{x_{1}}-f_{x_{2}}$ as a function of the halftone area coverages.

halftone and the decoder mask. The individual halftones can be represented in the form of a Fourier series as $h_{1}(x)=$ $\mu_{1}\left[1+2 \sum_{k_{1}=1}^{\infty} \operatorname{sinc}\left(k_{1} \mu_{1}\right) \cos \left(2 \pi k_{1} f_{x_{1}} x-2 \pi k_{1} d_{0}\right)\right]$ and $h_{2}(x)=\mu_{2}\left[1+2 \sum_{k_{2}=1}^{\infty} \operatorname{sinc}\left(k_{2} \mu_{2}\right) \cos \left(2 \pi k_{2} f_{x_{2}} x\right)\right]$. In the overlay of these halftones, the moire indexed by the pair $\left(k_{1}, k_{2}\right)$ is generated by the periodic function $2 \mu_{1} \mu_{2} \operatorname{sinc}\left(k_{1} \mu_{1}\right) \operatorname{sinc}\left(k_{2} \mu_{2}\right) \cos \left(2 \pi\left(k_{1} f_{x_{1}}-k_{2} f_{x_{2}}\right) x-2 \pi k_{1} d_{0}\right)$. The intensity of this moire is determined by its amplitude $2 \mu_{1} \mu_{2} \operatorname{sinc}\left(k_{1} \mu_{1}\right) \operatorname{sinc}\left(k_{2} \mu_{2}\right)$ that only depends on the constituent halftone area coverages $\left(\mu_{1}, \mu_{2}\right)$ and its index $\left(k_{1}, k_{2}\right)$. In practice, the individual screen frequencies $f_{x_{1}}$ and $f_{x_{2}}$ are large enough such that, except the $(1,1)$ moiré, the higher-order moirés have negligible amplitude due to the falloff of the sinc function. The periodic waveform for the $(1,1)$ moire is $2 \mu_{1} \mu_{2} \operatorname{sinc}\left(\mu_{1}\right) \operatorname{sinc}\left(\mu_{2}\right) \cos \left(2 \pi\left(f_{x_{1}}-f_{x_{2}}\right) x-2 \pi d_{0}\right)$ and one can readily see that the misalignment between the constituent halftones $d_{0}$ is observed as a change in the moiré phase and does not have an affect on its amplitude. Hence, in contrast to the transparency mask overlay based detection in the phase embedding method, careful control of the alignment of the decoder mask is not required for the detection of the watermark pattern in the frequency embedding. Fig. 14 shows the change in the moiré amplitude as a function of the halftone area coverages $\mu_{1}$ and $\mu_{2}$. It is clear that relatively large moiré amplitudes are achieved when the decoder mask have $50 \%$ area coverage $\left(\mu_{2}=0.5\right)$. Therefore, the decoder mask should have a reflectance around midtones to increase the detectability of the embedded watermark. 


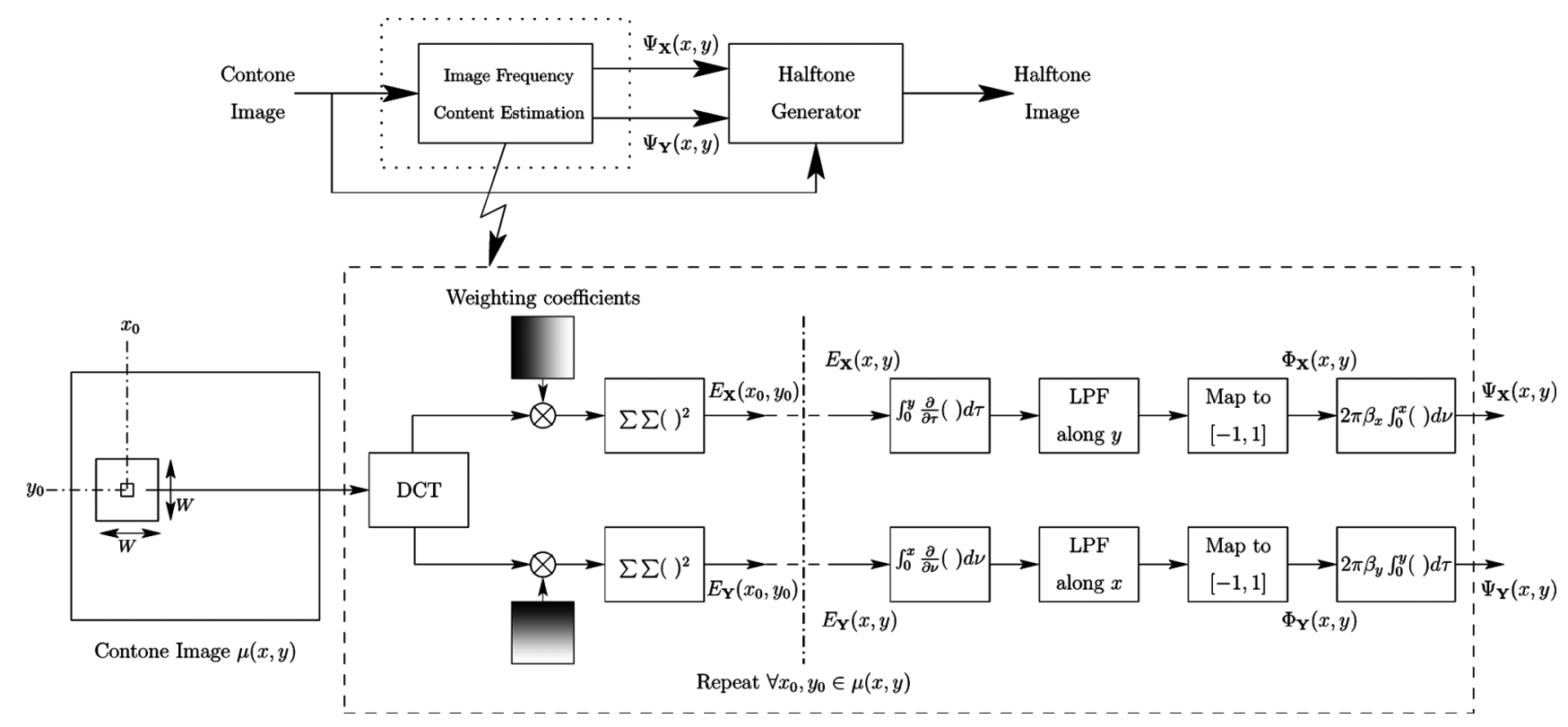

Fig. 15. Overview of self-modulated halftone generation.

This analysis can also be used to determine the detectability of the watermark. If the two levels of screen frequencies in the modulated halftone are $f_{x_{1}}$ and $f_{x_{1}}+\beta_{x}$, then the overlay of the modulated halftone with the decoder mask exhibit corresponding moiré patterns with frequencies $f_{x_{1}}-f_{x_{2}}$ and $f_{x_{1}}+\beta_{x}-f_{x_{2}}$. The screen frequency for the decoder mask $f_{x_{2}}$ takes an important role in the distinguishability of these moiré patterns. If $f_{x_{2}}$ is close to $f_{x_{1}}+\beta_{x} / 2$, then the two levels of the modulated image in the overlay exhibit moiré patterns with frequencies close to $\beta_{x} / 2$. Though the differences in moiré phase can be used to distinguish these patterns, observing the phase differences is practically challenging. Assume that the nonmodulated screen frequency is used for the decoder mask $\left(f_{x_{2}}=f_{x_{1}}\right)$, then a moiré pattern with frequency $\beta_{x}$ is observed in the regions with modified frequency and the other regions exhibit a change in their "DC response". The frequency deviation constant $\beta_{x}$ affects the detectability of the watermark for which the observation of at least one period of the moiré pattern is required. If $\beta_{x}$ is relatively small compared to screen frequency $f_{x_{1}}$, as indicated in Section III-C, total visible power does not increase significantly. However, this results in a low the embedding rate since a large distance is required to observe a single moiré period. On the other hand, a higher embedding rate can be achieved with a larger frequency deviation constant, which, however, can increase the perceptibility of the modulation in the halftone frequency. Hence, as we will describe in Section IV-C2, the frequency deviation constant should be chosen carefully to preserve the halftone image fidelity while maximizing the embedding rate.

\section{Self-Modulated Halftones}

As mentioned in Section I, the spatial and tonal rendering of the halftone image can be improved by adapting the halftone screen frequencies to the frequency content of the contone image. Self-modulated halftoning [2] provides a dynamic implementation of this approach by exploiting frequency modulation via CPM halftoning. An overview of self-modulated halftone generation is shown in Fig. 15. Recall that it is desirable for the halftone images to have a higher halftone frequency in image regions with high spatial activity (variation) and a lower halftone frequency in regions with lower spatial activity. We accomplish this by using the frequency modulating functions $\Phi_{\mathbf{X}}(x, y)$ and $\Phi_{\mathbf{Y}}(x, y)$ that are proportional to a local measure of spatial activity along horizontal and vertical directions, respectively. These are then utilized to obtain the phase modulation terms that modulate the screen frequencies in the threshold function as per (3). The halftone image is then obtained by comparing the frequency-modulated halftone threshold function against the contone image values using (1).

In Section III-B, we pointed out that in order to control the instantaneous frequencies along the horizontal/vertical directions, separability of the halftone threshold function is required. Since natural images are typically not separable, this requirement poses a difficulty for self-modulated halftones. The separability requirement may be slightly relaxed: if the phase modulation terms $\Psi_{\mathbf{X}}(x, y)$ and $\Psi_{\mathbf{Y}}(x, y)$ that are "locally constant" along the vertical and horizontal directions, respectively, the resulting threshold function in (3) can be "locally approximated" as a separable function. The instantaneous frequencies can then be approximated by replacing the total derivatives in (4) and (5) by the corresponding partial derivatives as

$$
\begin{aligned}
& f_{x}^{i}(x, y) \simeq f_{x}+\frac{1}{2 \pi} \frac{\partial \Psi_{\mathbf{X}}(x, y)}{\partial x} \text { and } \\
& f_{y}^{i}(x, y) \simeq f_{y}+\frac{1}{2 \pi} \frac{\partial \Psi_{\mathbf{Y}}(x, y)}{\partial y}
\end{aligned}
$$

respectively. Therefore, instead of pursuing complete separability, we ensure local constancy of the phase modulation terms $\Psi_{\mathbf{X}}(x, y)$ and $\Psi_{\mathbf{Y}}(x, y)$ along the vertical and horizontal directions, respectively.

In order to obtain the phase modulation terms $\Psi_{\mathbf{X}}(x, y)$ and $\Psi_{\mathbf{Y}}(x, y)$, we begin by first obtaining the estimates $E_{\mathbf{X}}(x, y)$ 


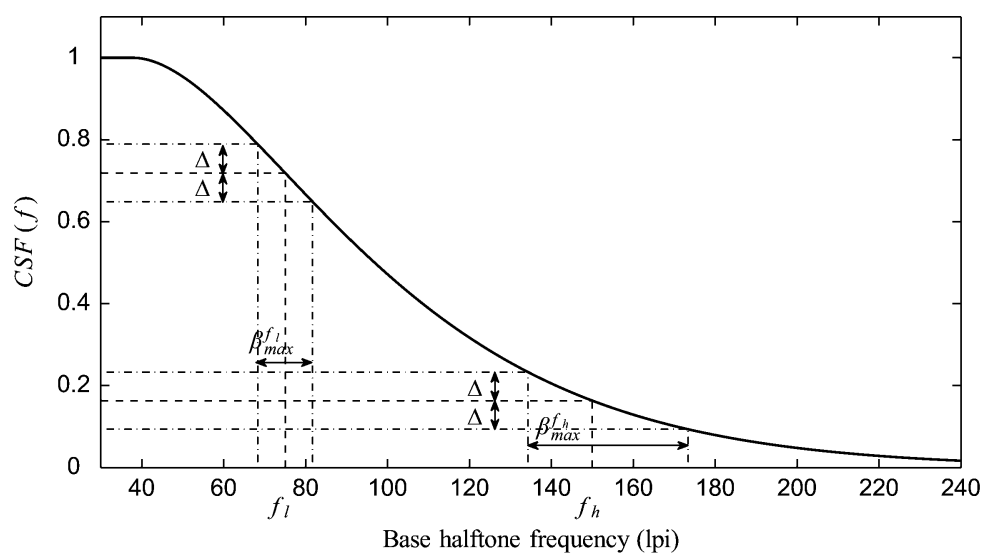

(a)

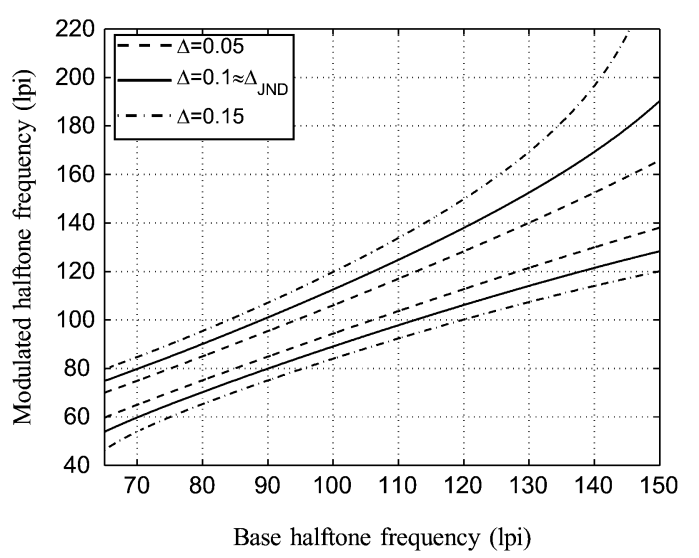

(b)

Fig. 16. (a) Computation of acceptable modulated halftone frequencies as a function of the base halftone frequency; (b) acceptable modulated halftone frequencies for $\Delta=0.05,0.1$, and 0.15 . The modulated halftone frequencies confined by these plots are perceptually acceptable with respect to the corresponding base halftone frequencies at the labeled tolerances.

and $E_{\mathbf{Y}}(x, y)$ of the local spatial activity in the horizontal and vertical directions, respectively. We describe the computation of $E_{\mathbf{X}}(x, y)$ (and of $\Psi_{\mathbf{X}}(x, y)$ ), the other term $E_{\mathbf{Y}}(x, y)$ (and $\left.\Psi_{\mathbf{Y}}(x, y)\right)$ can be obtained similarly. $E_{\mathbf{X}}\left(x_{0}, y_{0}\right)$ is computed as a weighted directional DCT energy estimate 5 at the location $\left(x_{0}, y_{0}\right)$. Two-dimensional Discrete Cosine Transform (DCT) coefficients are computed for a $W \times W$ window centered at $\left(x_{0}, y_{0}\right)$. The energy carried by the high-frequency DCT coefficients along the horizontal direction is a good indicator of the horizontal spatial activity within this window. Therefore, a frequency weighted squared-energy metric, where high frequencies are favored, is utilized to estimate the level of spatial activity. Specifically for this purpose we use a weighting function defined as $w[k]=1 / 2(1-\cos (\pi k / W))$ for $0 \leq k \leq W-1$, where $W$ is the length of the DCT window along the horizontal direction. Weightings are shown with shades of gray (ranging from black to white) in Fig. 15. The weighted DCT coefficients are squared and summed together to obtain an estimate of the spatial activity level along the horizontal direction at the location $\left(x_{0}, y_{0}\right)$.

Once the spatial activity level along the horizontal direction around each pixel in the image is estimated, the resulting function $E_{\mathbf{X}}(x, y)$ is used to build the modulating function $\Phi_{\mathbf{X}}(x, y)$ for the horizontal direction. The initial values of the modulating function can visible artifacts in the halftone image. Since the frequency modulation depends only on the partial derivative of the modulating function, the undesirable effect of the initial values can be eliminated by first taking the gradient and then the integral of $E_{\mathbf{X}}(x, y)$ along the vertical direction. Local constancy of the resulting signal along the vertical direction is (approximately) ensured by applying a low-pass filter in the vertical direction. The effect of the cut-off frequency of the low-pass filter on this approximation is discussed in Section IV-C1. The corresponding low-pass filtered signal is then normalized between $[-1,1]$ and used as the modulating function $\Phi_{\mathbf{X}}(x, y)$. The phase modulation term is finally generated as $\Psi_{\mathbf{X}}(x, y)=$ $2 \pi \beta_{x} \int_{0}^{x} \Phi_{\mathbf{X}}(\nu, y) d \nu$, where $\beta_{x}$ is the frequency deviation con-

${ }^{5}$ Here, we describe a specific realization based on the DCT. Alternate frequency domain representations, e.g., the DFT, may also be utilized instead of the DCT. stant. Consequently, the horizontal screen frequency is allowed to deviate between $f_{x} \pm \beta_{x}$ cpi. A discussion on the amount of modulation as a function of the base halftone frequency is presented in Section IV-C2.

1) Separability Issues: The (local) constancy of the frequency modulating function $\Phi_{\mathbf{X}}(x, y)$ along the vertical direction depends on the cut-off frequency of the low-pass filter (LPF). High cut-off frequency filters apply mild spatial blur that preserves the (local) spatial activity content of the image. The variations in the resulting frequency modulating function along the vertical direction, however, can compromise the accuracy of the approximation in (20) causing undesired variations as a byproduct. On the contrary, low cut-off frequency filters provide a modulating function $\Phi_{\mathbf{X}}(x, y)$ that exhibits greater local constancy along the vertical direction, thereby rendering the approximation in (20) more accurate. However, because the spatial blur corresponding to the LPF extends further in space along the vertical direction, $\Phi_{\mathbf{X}}(x, y)$ is influenced by spatial activity at regions that are more distant along the vertical direction, which is not desirable for the purpose of self-modulated halftoning. Thus, while the cut-off frequency of the low-pass filter should be low enough to ensure local constancy of the modulating functions, it should also be high enough to ensure that the modulating functions are representatives of the local frequency content in the image.

2) Perceptibility of Frequency-Modulated Halftones: The amount of modulation that is perceptually tolerable in frequency modulation based watermark embedding and self-modulated halftoning depends on the base halftone frequency. This can be seen with reference to Fig. 16(a), where the CSF of (17) is plotted with two base halftone frequencies $f_{l}$ and $f_{h}$, with $f_{l}<f_{h}$, are considered. Focusing on the case with a base halftone frequency $f_{l}$, as the halftone frequency is varied by frequency modulation, the contrast of the halftone texture also changes accordingly, where a contrast $C S F\left(f_{l}+\beta\right)$ is seen ${ }^{6}$ when the modulated frequency is $f_{l}+\beta$. The range of variation tolerable in the perceived contrast limits the maximum frequency deviation that is acceptable. For instance, in Fig. 16(a),

\footnotetext{
${ }^{6}$ Note in this process, we are approximating the halftone by the first harmonic component in the Fourier decomposition.
} 


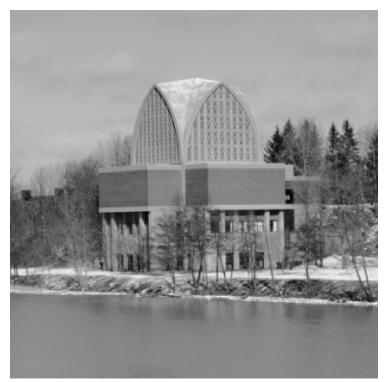

(a)

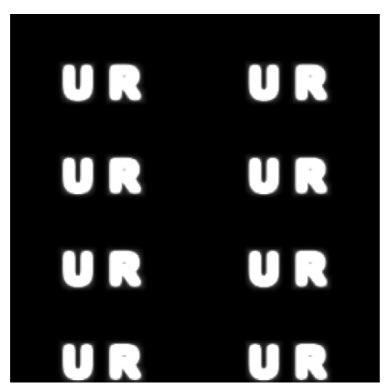

(b)

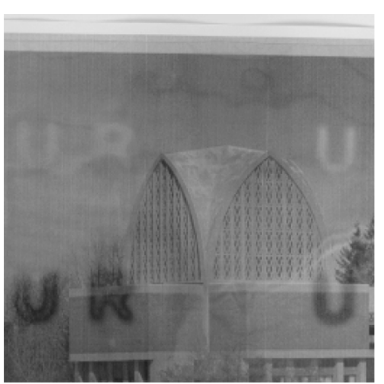

(c)

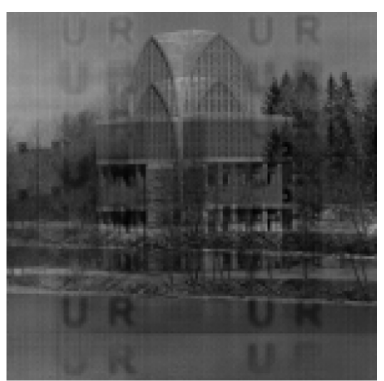

(d)

Fig. 17. Example of halftone watermarking using phase modulation: (a) shows the contone Chapel image and (b) shows the embedded watermark pattern; (c) and (d) show the detection results for the transparency mask overlay and self-referencing detectors, respectively. Embedded watermark pattern " $U R$ " is clearly visible for both detection methods.

the frequency deviation $\beta_{\max }^{f_{l}}$ is tolerable at the base halftone frequency $f_{l}$, where $\pm \Delta$ is the tolerable range in perceived contrast. A similar computation at $f_{h}$ [also shown in Fig. 16(a)] indicates that the corresponding maximum frequency deviation $\beta_{\max }^{f_{h}}$ is acceptable at a base halftone frequency $f_{h}$, where $\beta_{\text {max }}^{f_{h}}>\beta_{\text {max }}^{f_{l}}$.

In order to determine a just noticeable difference (JND) in contrast sensitivity $\Delta_{J N D}$, we conducted a psychovisual experiment that involved comparing the screen frequencies of nonmodulated ramps printed with 17 different halftone screen frequencies. From a viewing distance of approximately 12 inches, subjects viewed pairs of printed halftones, where the pair included a reference halftone with a screen frequency at approximately 71 lpi and a test halftone with a different screen frequency. As the test halftone was varied in frequency, each subject was asked to identify the sample for which the increase/decrease in halftone frequency over the reference was just noticeable. Once the pairs with just noticeable difference were identified, the perceptibility tolerance for each subject was computed by taking the difference between the contrast sensitivities of the corresponding halftone frequency pairs. Twelve subjects participated in this experiment and the average JND in contrast sensitivity were estimated as $\Delta_{J N D} \approx 0.106$. Fig. 16(b) shows the maximum and minimum acceptable modulated halftone frequencies as a function of the base halftone frequencies for different values of $\Delta$. In addition to the experimentally determined tolerance $\left(\Delta=0.1 \approx \Delta_{J N D}\right)$, we also included tolerances corresponding to oversensitive $(\Delta=0.05)$ and undersensitive ( $\Delta=0.15$ ) observers. Using these plots, one can determine the range of acceptable frequency deviation constants as a function of the base screen frequency in frequency modulation based watermark embedding and self-modulated halftoning.

\section{EXPERIMENTAL RESULTS}

An experimental evaluation of continuous phase modulation was conducted for the proposed applications. Our setup utilized an electrophotographic printer with an addressability of $1200 \times 1200 \mathrm{dpi}$. Screen frequencies $f_{x}$ and $f_{y}$ were chosen to be 50 cycles per inch (cpi), which corresponds to a base halftone frequency of $50 \sqrt{2} \simeq 71$ lines per inch (lpi). CPM halftones were generated for the two applications that we described in Section IV. All images were printed in an 8 in $\times 8$ in format in the experiments. Since the experimental performance is best assessed visually, we present visual results for one example image for each of the applications. As should be self-evident, the methods are general and applicable to most image content.

\section{A. Halftone Watermarking Using Phase Modulation}

Experimental performance of watermark pattern embedding via continuous phase modulation is investigated first. We illustrate the operation of the proposed methodology by using the Chapel image shown in Fig. 17(a). For halftone watermarking using phase modulation, we embedded the self-referencing detector compatible watermark pattern shown in Fig. 17(b) with $U R$ letters representing University of Rochester. Maximum phase deviation of $\kappa=\pi$ radians was utilized. The CPM halftone was printed on our test printer and the embedded watermark pattern was detected using both the transparency overlay and self-referencing detectors. First, a decoder mask consisting of nonmodulated halftone for a halftone area coverage of $50 \%$ was printed on a transparency and overlaid on the modulated halftone. Fig. 17(c) shows a portion of the resulting overlay captured with a digital camera. Next, the printed CPM image was scanned with a flatbed scanner with optical resolution of $1200 \times 1200$ dpi and using the self-referencing detection method described in Section IV-A the resulting image in Fig. 17(d) is obtained. The watermark pattern "UR" is clearly visible in the resulting overlays of both detectors. As expected, the marks are more visible in midtone gray levels. Furthermore, as anticipated by our analysis of Section IV-A1 (see Fig. 12), due to variation in the registration between the decoder mask and the CPM watermarked halftone, the detected watermark regions in Fig. 17(c) also vary: being either lighter or darker than the background or being visible only in the transition edge regions. Fig. 18 demonstrates the effect of registration between the two halftones on the detected watermark for initial misregistrations $d_{0}=0,0.25$, and 0.5 in halftone cell units. As previously discussed in Fig. 12, the recovered watermark pattern corresponding to these misregistrations is either a scaled version of the modulation pattern [Fig. 18(c)], an inverted version [Fig. 18(a)], or a version in which only the boundaries corresponding to the transitions is seen [Fig. 18(b)]. Note that the self-referencing detection does not suffer from this effect since the scanned image itself is used as the decoder mask. Both detection methods are invariant to translations of the CPM image. Tests reveal that the watermark is detectable for rotations up-to $5^{\circ}$ in the printing/scanning process. 


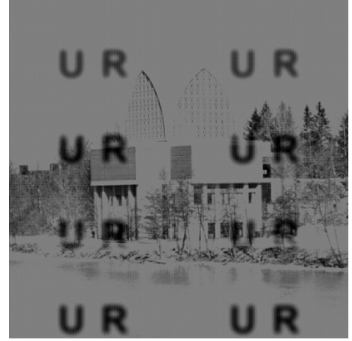

(a) $d_{0}=0$

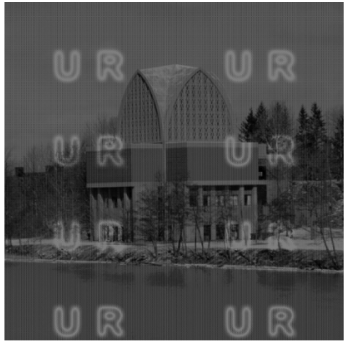

(b) $d_{0}=0.25$

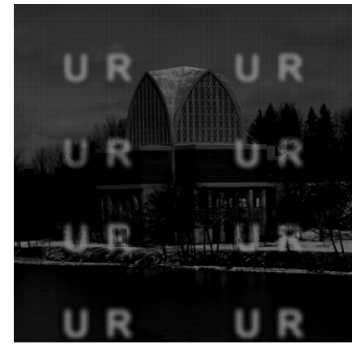

(c) $d_{0}=0.5$

Fig. 18. Digital simulation results showing the spatial dependence of the overlay reflectance on the modulating signal and the initial misregistration. (a), (b), and (c) show demodulated watermark patterns for misregistration displacements $d_{0}=0,0.25$, and 0.5 , respectively.

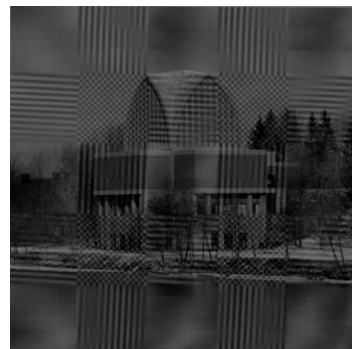

(a)

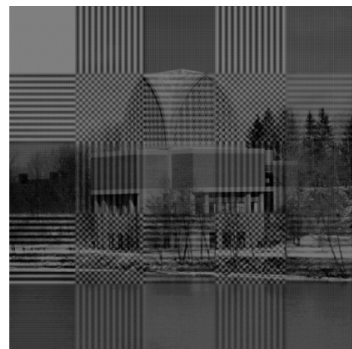

(b) $d_{0}=0$

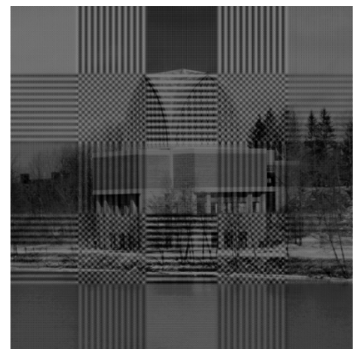

(c) $d_{0}=0.25$

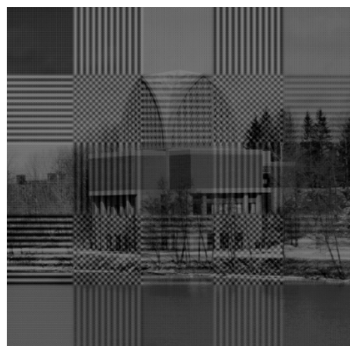

(d) $d_{0}=0.5$

Fig. 19. Detection results of the watermark embedded via frequency modulation: (a) shows the detection result using the scanned image; (b), (c), and (d) show the digital simulation results using digital halftones and for the initial misregistration displacements $d_{0}=0,0.25$, and 0.5 , respectively.

\section{B. Halftone Watermarking Using Frequency Modulation}

Next, we consider embedding binary watermark patterns along the horizontal and vertical directions by modulating the halftone screen frequencies. For demonstration purposes, the watermark pattern $\{0,1,0,1,0\}$ is embedded along both directions. Maximum screen frequency deviations of $\beta_{x}=10$ and $\beta_{y}=10 \mathrm{cpi}$ were utilized for the frequency modulation embedding. After printing/scanning the CPM halftone, we digitally superimposed the scanned image with a decoder mask with $50 \%$ halftone area coverage generated at the base halftone frequency (and orientation). Fig. 19(a) shows the resultant overlay. The moiré patterns along horizontal/vertical directions are clearly visible, from which the embedded watermark pattern can be easily detected.

In addition, in order to demonstrate the invariance of the detection to the registration between the modulated halftone and the decoder mask, we digitally simulated the overlay of the digital halftones with the decoder mask for misregistration displacements $d_{0}=0,0.25$, and 0.5 between the two images in Fig. 19(b)-(d), respectively. As predicted by the analysis of Section IV-B1, we see that in this case the appearance of the moiré pattern is not impacted by misregistration. Another advantage in the frequency embedding method is that the technique is considerably more robust to angular rotation in the detection process (at least for visual detection) since the detected pattern is observed as moiré. As a matter of fact Fig. 19(a) exhibits an example of this property. Although the scanned image has slight angular rotation (observe the angular orientation of the moiré lines), the embedded watermark pattern can still be recovered. This can be attractive in applications where rapid detection of the watermark may be required (for instance by scanning with a hand-held scanner).

\section{Self-Modulated Halftones}

For the demonstration of self-modulated halftoning, we use the contone Library image shown in Fig. 20(a). The DCT window size used for estimating the local spatial activity was chosen as $W=1 \mathrm{~cm}$ and the spatial activity estimates $E_{\mathbf{X}}(x, y)$ and $E_{\mathbf{Y}}(x, y)$ for the image are shown in Fig. 20(b) and (c), respectively. In order to ensure local constancy of the modulating functions $\Phi_{\mathbf{X}}(x, y)$ and $\Phi_{\mathbf{Y}}(x, y)$ (see Section IV-C1), we use a spatial Gaussian low-pass filter. Through empirical evaluation the variance of the Gaussian was chosen to correspond to a $1.5 \mathrm{~cm}$ length and frequency deviation constants $\beta_{x}$ and $\beta_{y}$ were chosen as $10 \mathrm{cpi}$. The printed/scanned self-modulated Library halftone is shown in Fig. 21(a). Due to downsampling of the image to fit in this document, the printed version of this document may not be appropriate for evaluation, however, the actual effect can be seen on the computer screen (by zooming in). Fig. 21(b) and (c) show enlarged segments from the roof of the building and sky to illustrate the effect of frequency modulation in high and low spatial activity regions, respectively. From these figures it can be seen that the self-modulation indeed causes a significant frequency variation in the halftone. Full-resolution prints also illustrate that the method alleviates the tonal versus spatial resolution trade-off.

Note that in the presence of frequency modulation, a single halftone periodic cell is absent and effectively the "cell size" varies with frequency. The interaction between the varying halftone frequency and the printing grid can cause moire patterns if the printing resolution is insufficient. At high enough printing resolution, however, this moiré is minimal and can be further mitigated in printing systems through the use of high addressability [5]. 


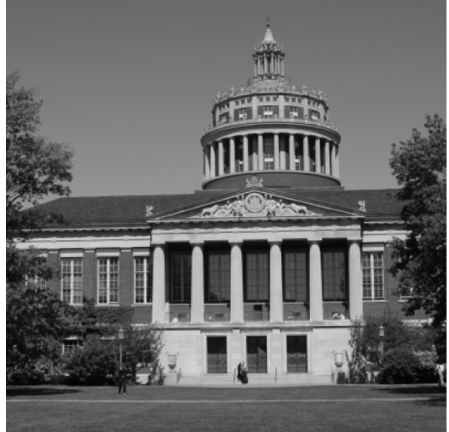

(a) Contone Library image

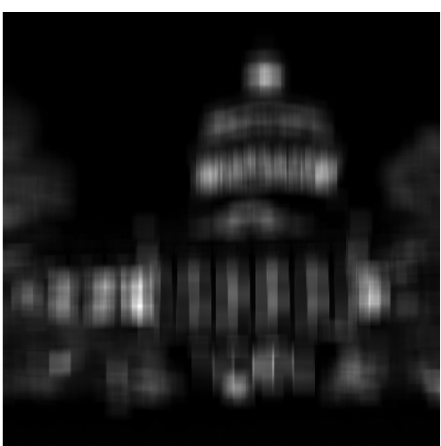

(b) $E_{\mathbf{X}}(x, y)$

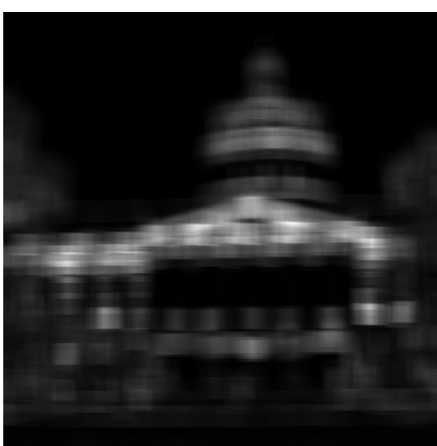

(c) $E_{\mathbf{Y}}(x, y)$

Fig. 20. Contone Library image and the corresponding spatial activity representations along the horizontal and vertical directions.

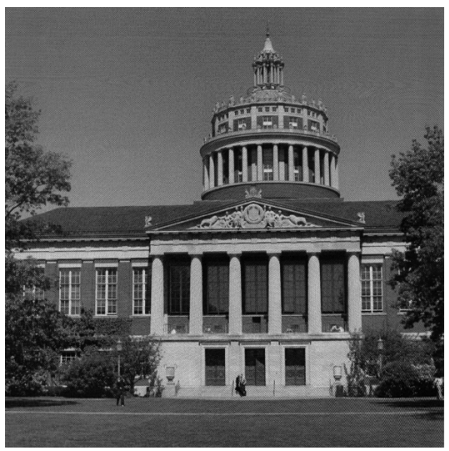

(a) Self-modulated Library halftone

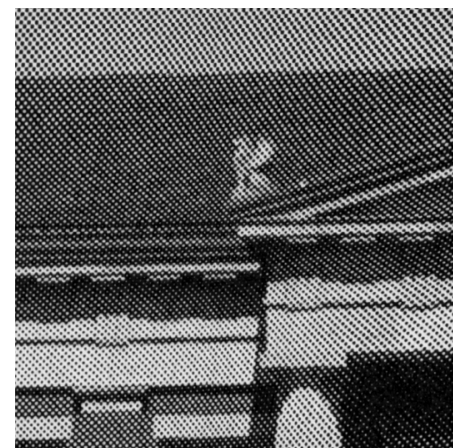

(b) Note the high screen frequencies (closely spaced halftone spots) in lower half of the image

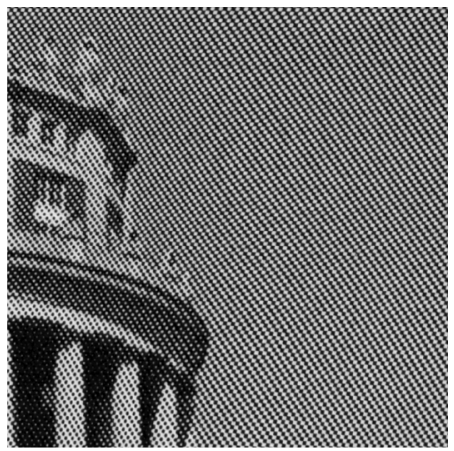

(c) Note the low screen frequencies (coarsely spaced halftone spots) in the upper right corner

Fig. 21. Self-modulated Library halftone. Enlarged views of high and low spatial activity regions are shown in (b) and (c), respectively.

\section{CONCLUSION}

Continuous phase-modulated (CPM) halftones are a generalization of the periodic clustered-dot halftones that allows controlled variations in halftone phase in different regions of the printed page. The method uses an analytic halftone threshold function whose phase can be modulated independently using an auxiliary signal, thereby providing the flexibility of dynamic control over the halftone phase during the halftoning process. CPM halftoning can accomplish either phase or frequency modulation enabling applications in watermarking and in content adaptive halftoning. Experimental results demonstrate the efficacy of CPM halftones for both applications. Spectral analysis of CPM halftones presented in this paper can be utilized to assess the visibility of changes introduced by the phase/frequency modulation.

\section{APPENDIX A}

\section{FOURIER SERIES EXPANSION OF PULSE-POSITION} MOdULATED HALFTONE

Modulating signal $\varepsilon_{n}$ is a periodic function with period $N$. Hence, $h_{P M}(x)$ is a periodic function with period $N \tau$ and it can be represented in terms of its Fourier series [42] as

$$
\begin{aligned}
h_{P M}(x)= & a_{0_{P M}} \\
& +2 \sum_{k=1}^{\infty}\left[a_{k_{P M}} \cos \left(\frac{2 \pi k}{N \tau} x\right)+b_{k_{P M}} \sin \left(\frac{2 \pi k}{N \tau} x\right)\right]
\end{aligned}
$$

where the coefficient at DC is $a_{0 P M}=1 / N \tau \int_{0}^{N \tau} h_{P M}(x) d x=$ $\tau_{p} / \tau$, and the coefficients at frequency $k / N \tau$ are

$$
\begin{aligned}
a_{k_{P M}} & =\frac{1}{N \tau} \int_{0}^{N \tau} h_{P M}(x) \cos \left(\frac{2 \pi k}{N \tau} x\right) d x \\
& =\frac{1}{N \tau} \int_{0}^{N \tau} \sum_{n=0}^{N-1} \operatorname{rect}\left(\frac{x-n \tau-\varepsilon_{n}-\frac{\tau}{2}}{\tau_{p}}\right) \cos \left(\frac{2 \pi k}{N \tau} x\right) d x \\
& =\left.\frac{1}{2 \pi k} \sum_{n=0}^{N-1} \sin \left(\frac{2 \pi k}{N \tau} x\right)\right|_{n \tau+\varepsilon_{n}+\tau / 2-\tau_{p} / 2} ^{n \tau+\varepsilon_{n}+\tau / 2+\tau_{p} / 2} \\
& =\frac{\tau_{p}}{N \tau} \operatorname{sinc}\left(\frac{k \tau_{p}}{N \tau}\right) \sum_{n=0}^{N-1} \cos \left(\frac{2 \pi k}{N \tau}\left(n \tau+\varepsilon_{n}+\frac{\tau}{2}\right)\right)
\end{aligned}
$$

and similarly

$$
\begin{aligned}
b_{k_{P M}} & =\frac{1}{N \tau} \int_{0}^{N \tau} h_{P M}(x) \sin \left(\frac{2 \pi k}{N \tau} x\right) d x \\
& =\left.\frac{1}{2 \pi k} \sum_{n=0}^{N-1} \cos \left(\frac{2 \pi k}{N \tau} x\right)\right|_{n \tau+\varepsilon_{n}+\tau / 2-\tau_{p} / 2} ^{n \tau+\varepsilon_{n}+\tau / 2+\tau_{p} / 2} \\
& =\frac{\tau_{p}}{N \tau} \operatorname{sinc}\left(\frac{k \tau_{p}}{N \tau}\right) \sum_{n=0}^{N-1} \sin \left(\frac{2 \pi k}{N \tau}\left(n \tau+\varepsilon_{n}+\frac{\tau}{2}\right)\right) .
\end{aligned}
$$




\section{APPENDIX B}

\section{FOURIER SERIES EXPANSION OF PULSE-FREQUENCY-WIDTH} MOdUlated HALFTONE

A period of $h_{F M}(x)$ is of length $\sum_{n=0}^{N-1} \tau_{n}$ and it can be shown that

$$
\begin{aligned}
\sum_{n=0}^{N-1} \tau_{n}= & \sum_{n=0}^{N-1} \tau\left(1+\alpha \sin \left(\frac{2 \pi n}{N}+\phi\right)\right) \\
= & N \tau+\frac{1}{2 j} \alpha \tau \\
& \times \sum_{n=0}^{N-1}\left(e^{j \phi} e^{j 2 \pi n / N}-e^{-j \phi} e^{-j 2 \pi n / N}\right) \\
= & N \tau+\frac{1}{2 j} \alpha \tau \\
& \times\left(e^{j \phi} \frac{1-e^{j 2 \pi N / N}}{1-e^{j 2 \pi / N}}-e^{-j \phi} \frac{1-e^{-j 2 \pi N / N}}{1-e^{-j 2 \pi / N}}\right) \\
= & N \tau .
\end{aligned}
$$

Hence, the halftone can be represented in terms of its Fourier series [42] as

$$
\begin{aligned}
h_{F M}(x)= & a_{0_{F M}} \\
& +2 \sum_{k=1}^{\infty}\left[a_{k_{F M}} \cos \left(\frac{2 \pi k}{N \tau} x\right)+b_{k_{F M}} \sin \left(\frac{2 \pi k}{N \tau} x\right)\right]
\end{aligned}
$$

where the coefficient at DC is $a_{0_{F M}}=1 / N \tau \int_{0}^{N \tau} h_{F M}(x) d x=$ $\tau_{p} / \tau$ and the coefficients at frequency $k / N \tau$ are

$$
\begin{aligned}
a_{k_{F M}} & =\frac{1}{N \tau} \int_{0}^{N \tau} h_{F M}(x) \cos \left(\frac{2 \pi k}{N \tau} x\right) d x \\
& =\frac{1}{N \tau} \int_{0}^{N \tau} \sum_{n=0}^{N-1} \operatorname{rect}\left(\frac{x-\sum_{m=0}^{n-1} \tau_{m}-\frac{\tau_{n}}{2}}{\tau_{p, n}}\right) \cos \left(\frac{2 \pi k}{N \tau} x\right) d x \\
& =\left.\frac{1}{2 \pi k} \sum_{n=0}^{N-1} \sin \left(\frac{2 \pi k}{N \tau} x\right)\right|_{\sum_{m=0}^{n-1} \tau_{m}+\tau_{n} / 2-\tau_{p, n} / 2} ^{\sum_{m=1}^{n-1} \tau_{m}+\tau_{n} / 2+\tau_{p, n} / 2} \\
& =\sum_{n=0}^{N-1} \frac{\tau_{p, n}}{N \tau} \operatorname{sinc}\left(\frac{k \tau_{p, n}}{N \tau}\right) \cos \left(\frac{2 \pi k}{N \tau}\left(\sum_{m=0}^{n-1} \tau_{m}+\frac{\tau_{n}}{2}\right)\right)
\end{aligned}
$$

and similarly

$$
\begin{aligned}
b_{k_{F M}} & =\frac{1}{N \tau} \int_{0}^{N \tau} h_{F M}(x) \sin \left(\frac{2 \pi k}{N \tau} x\right) d x \\
& =\left.\frac{1}{2 \pi k} \sum_{n=0}^{N-1} \cos \left(\frac{2 \pi k}{N \tau} x\right)\right|_{\sum_{m=0}^{n-1} \tau_{m}+\tau_{n} / 2-\tau_{p, n} / 2} ^{\sum_{m=0}^{n-1} \tau_{m}+\tau_{n} / 2+\tau_{p, n} / 2} \\
& =\sum_{n=0}^{N-1} \frac{\tau_{p, n}}{N \tau} \operatorname{sinc}\left(\frac{k \tau_{p, n}}{N \tau}\right) \sin \left(\frac{2 \pi k}{N \tau}\left(\sum_{m=0}^{n-1} \tau_{m}+\frac{\tau_{n}}{2}\right)\right) .
\end{aligned}
$$

\section{ACKNOWLEDGMENT}

The authors would like to thank Dr. R. P. Loce of Xerox Research Center Webster, Webster, NY, for discussions, suggestions, and help with printing high-resolution images.

\section{REFERENCES}

[1] B. Oztan and G. Sharma, "Continuous phase modulated halftones and their application to halftone data embedding," in Proc. IEEE Int. Conf. Acoustics Speech and Signal Processing, May 2006, vol. II, pp. 333-336.

[2] B. Oztan and G. Sharma, "Self-modulated halftones," in Proc. IEEE Int. Conf. Image Processing, Oct. 2006, pp. 1533-1536.

[3] J. C. Stoffel and J. F. Moreland, "A survey of electronic techniques for pictoral image reproduction," IEEE Trans. Commun., vol. COM-29, no. 12, pp. 1898-1925, Dec. 1981

[4] P. R. Jones, "Evolution of halftoning technology in the United States patent literature," J. Electron. Imag., vol. 3, no. 3, pp. 257-275, Jul. 1994.

[5] C. M. Hains, S. Wang, and K. T. Knox, "Digital color halftones," in Digital Color Imaging Handbook, G. Sharma, Ed. Boca Raton, FL: CRC, 2003, ch. 6.

[6] W. H. F. Talbot, "Improvements in the Art of Engraving," British Patent Spec. No. 565, Oct. 29, 1852.

[7] J. Proakis and M. Salehi, Fundamentals of Communication Systems. Upper Saddle River, NJ: Prentice-Hall, 2005.

[8] R. L. van Renesse, Optical Document Security, 3 ed. Boston, MA: Artech House, 2005

[9] C. M. Briquet, Les Filigranes: Dictionaire Histoire Des Marques Due Papier Des Leur Appartion Vers 1282, Jusquen 1600 2nd ed., Leipzig, 1923.

[10] P. W. Wong and N. D. Memon, "Image processing for halftones," IEEE Signal Process. Mag., vol. 20, no. 4, pp. 59-70, Jul. 2003.

[11] I. Amidror, S. Chosson, and R. D. Hersch, "Moiré methods for the protection of documents and products: A short survey," in Proc. J. Phys. Conf. Ser., 2007, vol. 77, p. 012001.

[12] Z. Baharav and D. Shaked, "Watermarking of dither halftoned images," in Proc. SPIE: Security and Watermarking of Multimedia Contents, $\mathrm{P}$ W. Wong and E. J. Delp, Eds., 1999, vol. 3657, pp. 307-316.

[13] H. Z. Hel-Or, "Watermarking and copyright labeling of printed images," J. Electron. Imag., vol. 10, no. 3, pp. 794-803, Jul. 2001.

[14] I. Amidror, "New print-based security strategy for the protection of valuable documents and products using moiré intensity profiles," in Proc. SPIE: Optical Security and Counterfeit Deterrence Technique IV, R. L. van Renesse, Ed., 2002, vol. 4677, pp. 89-100.

[15] C. Liu, S. Wang, and B. Xu, "Authenticate your digital prints with glossmark images," in Proc. IS\&T NIP20: Int. Conf. Digital Printing Technologies, Oct. 2004, pp. 312-316.

[16] O. Bulan, V. Monga, G. Sharma, and B. Oztan, "Data embedding in hardcopy images via halftone-dot orientation modulation," in Proc. SPIE: Security, Forensics, Steganography, and Watermarking of Multimedia Contents X, E. J. Delp, P. W. Wong, J. Dittmann, and N. D. Memon, Eds., vol. 6819, pp. 68190C-1-12.

[17] V. Ostromoukhov, N. Rudaz, I. Amidror, P. Emmel, and R. D. Hersch, "Anti-counterfeiting feature of artistic screening," in Proc. SPIE: Holographic and Diffractive Techniques, G. J. Dausmann, Ed., 1996, vol 2951, pp. 126-133.

[18] S. Wang, "Stoclustic (stochastic clustered) halftone screen design," in Proc. IS\&T's NIP 13: Int. Conf. Digital Printing Technologies, Seattle, WA, Nov. 1997, pp. 516-521.

[19] S. Wang and K. T. Knox, "Embedding digital watermarks in halftone screens," in Proc. SPIE: Security and Watermarking of Multimedia Contents II, P. W. Wong and E. J. Delp, Eds., 2000, vol. 3971, pp. 218-227.

[20] S. Wang, "Digital Watermarking Using Phase-Shifted Stoclustic Screens," U.S. Patent 6,252,971, 2001.

[21] G. Sharma and S. Wang, "Show-through watermarking of duplex printed documents," in Proc. SPIE: Security, Steganography, and Watermarking of Multimedia Contents VI, E. J. Delp and P. W. Wong, Eds., 2004, vol. 5306, pp. 670-684.

[22] S. Huang and J. K. Wu, "Optical watermarking for printed documen authentication," IEEE Trans. Inf. Forensics Security, vol. 2, no. 2, pp. 164-173, Jun. 2007.

[23] O. Bryngdahl, "Halftone images: Spatial resolution and tone reproduction," J. Opt. Soc. Amer., vol. 68, no. 3, pp. 416-422, Mar. 1977.

[24] K. T. Knox, "Digital halftoning algorithms and parameters," in Proc. Int. Conf. LASERS, 1990, pp. 619-625.

[25] P. G. Roetling, "Halftone method with edge enhancement and moire supression," J. Opt. Soc. Amer., vol. 66, no. 10, pp. 985-989, Oct. 1976.

[26] T. S. Rao and G. R. Arce, "Halftone patterns for arbitrary screen periodicities," J. Opt. Soc. Amer. A, vol. 5, no. 9, pp. 1502-1511, Sep. 1988.

[27] Z. Fan, "Dot-to-dot error diffusion," J. Electron. Imag., vol. 2, no. 1, pp. 62-66, Jan. 1993

[28] L. Velho and J. Gomes, "Stochastic screening dithering with adaptive clustering," Proc. ACM SIGGRAPH, pp. 273-276, 1995. 
[29] Y. Zhang, "Adaptive ordered dither," CVGIP: Graph. Models Image Process., vol. 59, no. 1, pp. 49-53, Jan. 1997.

[30] H. Z. Hel-Or, X. M. Zhang, and B. A. Wandell, "Adaptive cluster dot dithering," J. Electron. Imag., vol. 8, no. 2, pp. 133-144, Apr. 1999.

[31] D. N. Curry, "Analytic clustered halftone dots," in Proc. SPIE: Color Imaging: Device Independent Color, Color Hardcopy, and Graphic Arts II, G. B. Beretta and R. Eschbach, Eds., 1997, vol. 3018, pp. 323-330.

[32] R. J. Pellar and L. Green, "Electronic Halftone Generator," U.S. Patent 4149183, 1979.

[33] R. J. Pellar, "Electronic Halftone Generator," U.S. Patent 4196451, 1980.

[34] J. B. Anderson, T. Aulin, and C.-E Sundberg, Digital Phase Modulation, 1 ed. New York: Plenum, 1986

[35] R. Näsänen, "Visibility of halftone dot textures," IEEE Trans. Syst., Man, Cybern., vol. 14, no. 6, pp. 920-924, Jun. 1984.

[36] H. Kaufman and E. King, "Spectral power density functions in pulse time modulation," IEEE Trans. Inf. Theory, vol. 1, no. 1, pp. 40-46, Mar. 1955.

[37] J. R. Alford and T. Mitsa, "Qualitative comparison of visual models in an iterative halftoning procedure," in Proc. IEEE Intl. Conf. Image Processing, Oct. 1995, vol. II, pp. 339-342.

[38] T. Mitsa, K. Varkur, and J. Alford, "Frequency-channel-based visual models as quantitative quality measures in halftoning," in Proc. SPIE: Human Vision, Visual Processing, and Digital Display IV, J. P. Allebach and B. E. Rogowitz, Eds., 1993, vol. 1913, pp. 390-401.

[39] J. L. Mannos and D. J. Sakrison, "The effects of a visual fidelity criterion on the encoding of images," IEEE Trans. Inf. Theory, vol. IT-20, no. 4, pp. 525-536, Jul. 1974.

[40] B. Oztan, G. Sharma, and R. P. Loce, "Misregistration sensitivity in clustered-dot color halftones," J. Electron. Imag., vol. 17, no. 2, pp. 023004,1-30, Apr./Jun. 2008

[41] I. Amidror, R. D. Hersch, and V. Ostromoukhov, "Spectral analysis and minimization of moiré patterns in color separation," J. Electron. Imag., vol. 3, no. 3, pp. 295-317, Jul. 1994.

[42] R. N. Bracewell, The Fourier Transform and Its Applications, 2nd ed. New York: McGraw-Hill, 1986.

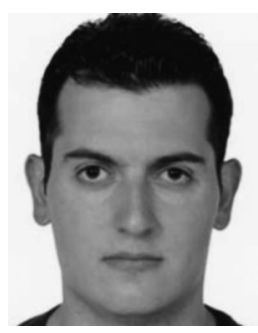

Basak Oztan (S'00) received the B.S. degree with high honors in electrical and electronics engineering from the Middle East Technical University, Ankara, Turkey, in 2003, and the M.S. degree in electrical and computer engineering from University of Rochester, Rochester, NY, in 2004. He is currently pursuing the Ph.D. degree in the Department of Electrical and Computer Engineering, University of Rochester.

He was with Xerox Webster Research Center, Webster, NY, during the summers of 2005 and 2006 as a research intern. His research interests include color imaging, color halftoning, and multimedia security.
Mr. Oztan is a student member of the IEEE Signal Processing Society, SPIE, and IS\&T. He serves as a reviewer for the IEEE TRANSACTIONS ON IMAge Processing, the IEEE TRANSACTIONS ON INFORMATION ForENSICS AND SECURITY, the IEEE Signal PRocessing LeTters, and the SPIE/IS\&T Journal of Electronic Imaging. He was the recipient of the student paper award at 2006 IEEE International on Conference on Acoustics, Speech, and Signal Processing (ICASSP) in the image and multidimensional signal processing category.

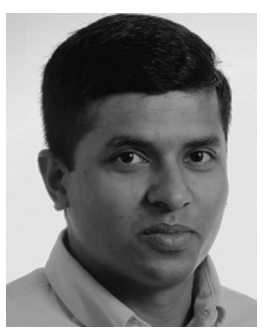

Gaurav Sharma (SM'00) received the B.E. degree in electronics and communication engineering from Indian Institute of Technology Roorkee (formerly University of Roorkee), India, in 1990; the M.E. degree in electrical communication engineering from the Indian Institute of Science, Bangalore, India, in 1992; and the M.S. degree in applied mathematics and the Ph.D. degree in electrical and computer engineering from North Carolina State University, Raleigh, in 1995 and 1996, respectively.

From August 1992 through August 1996, he was a research assistant at the Center for Advanced Computing and Communications, ECE Department, NCSU. From August 1996 through August 2003, he was with Xerox Research and Technology, Webster, NY, initially as a member of research staff and subsequently at the position of principal scientist. Since Fall 2003, he is an Associate Professor at the University of Rochester in the Department of Electrical and Computer Engineering and in the Department of Biostatistics and Computational Biology. He is also the Director for the Center for Electronic Imaging Systems (CEIS), a New York state funded center for promoting joint university-industry research and technology development, which is housed at the University of Rochester. His research interests include multimedia security and watermarking, color science and imaging, genomic signal processing, and image processing for visual sensor networks. He is the editor of the Color Imaging Handbook (CRC, 2003).

Dr. Sharma's is a member of Sigma Xi, Phi Kappa Phi, Pi Mu Epsilon, IS\&T, and the signal processing and communications societies of the IEEE. He was the 2007 chair for the Rochester section of the IEEE and served as the 2003 chair for the Rochester chapter of the IEEE Signal Processing Society. He currently serves as the vice chair for the IEEE Signal Processing Society's Image and multidimensional signal processing (IMDSP) technical committee and is an advisory member of the IEEE Standing committee on Industry DSP. He currently serves as an associate editor the Journal of Electronic Imaging and in the past has served as an associate editor for the IEEE TRANSACTIONS ON IMAGE PROCESSING and the IEEE TRANSACTIONS ON INFORMATION FORENSICS AND SECURITY. 